\title{
Zn(II)/Cd(II) Terephthalate Coordination Polymers Incorporating Bi-, Tri- and Tetratopic Phenylamine Derivatives: Crystal Structures and Photoluminescent Properties
}

Zhenzhen Shi, ${ }^{\dagger}$ Zhaorui Pan,,$^{\dagger, *}$ Hailang Jia, ${ }^{\dagger}$ Shuguang Chen, ${ }^{\dagger}$ Ling Qin, ${ }^{\dagger}$ Hegen Zheng ${ }^{*} \dagger$

${ }^{\dagger}$ State Key Laboratory of Coordination Chemistry, School of Chemistry and Chemical Engineering, Collaborative Innovation Center of Advanced Microstructures, Nanjing University, Nanjing 210093, P. R. China

${ }^{\ddagger}$ School of Environmental Science, Nanjing Xiaozhuang University, Nanjing 211171, P. R. China

Fax: (+86)25-83314502, E-mail: zhenghg@ nju.edu.cn 


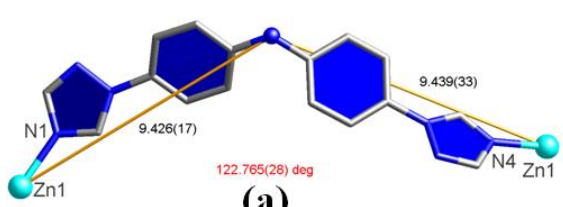

(a)

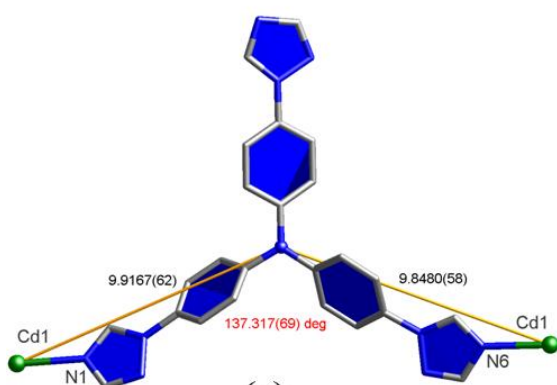

(c)
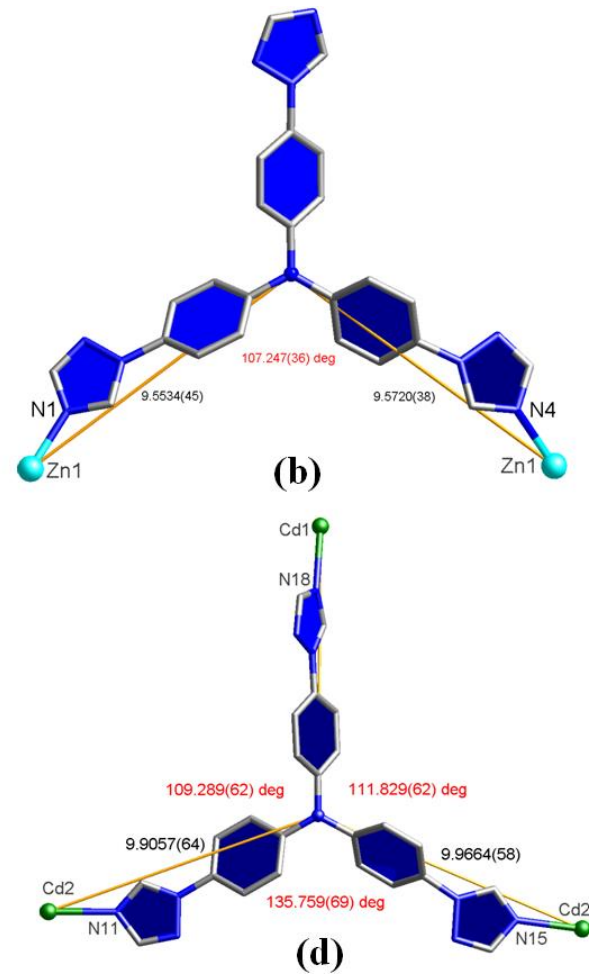

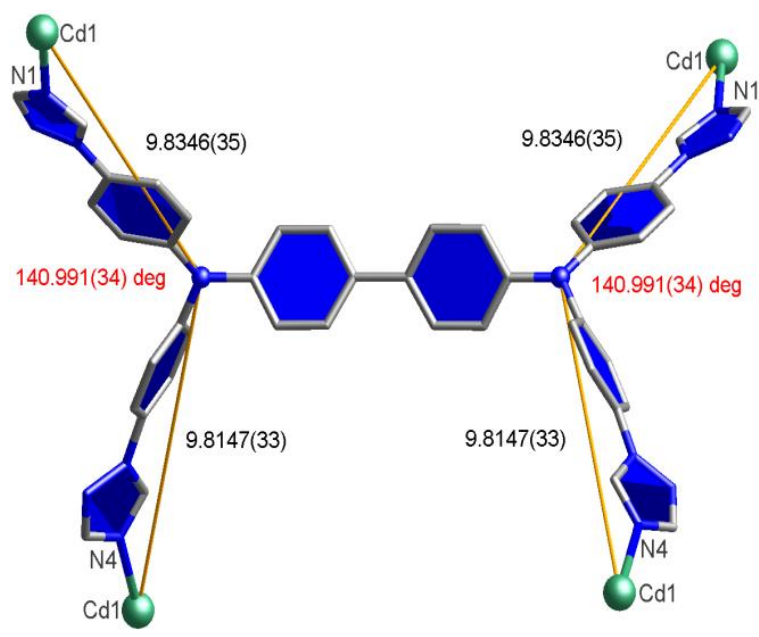

(e)

Figure S1. The conformation of BTPA, TTPA and TTPBDA ligands in complexes 1-4

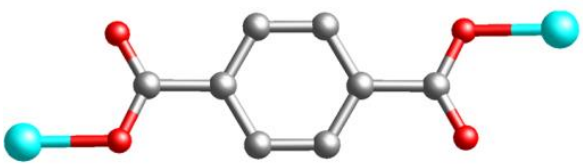

(a)

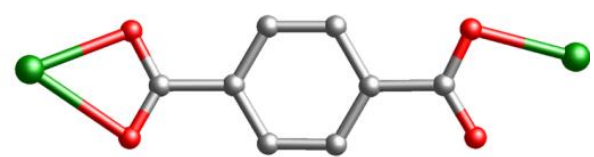

(b)

Figure S2. The coordination modes of TPA ${ }^{2-}$ ligand in complexes 1-4 
(a)

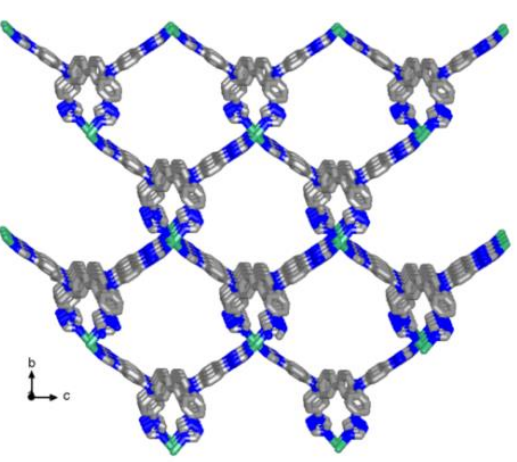

(c)

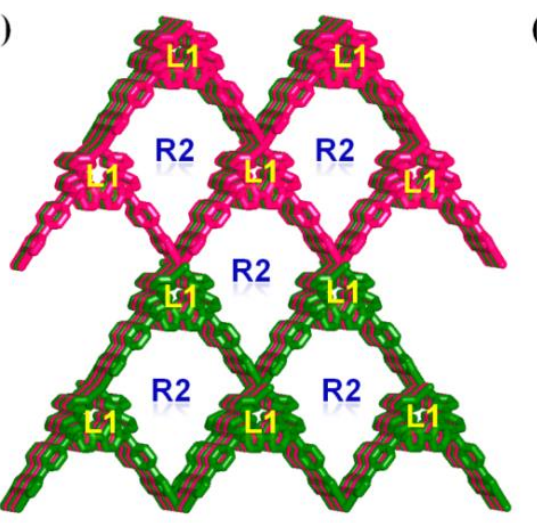

(b)

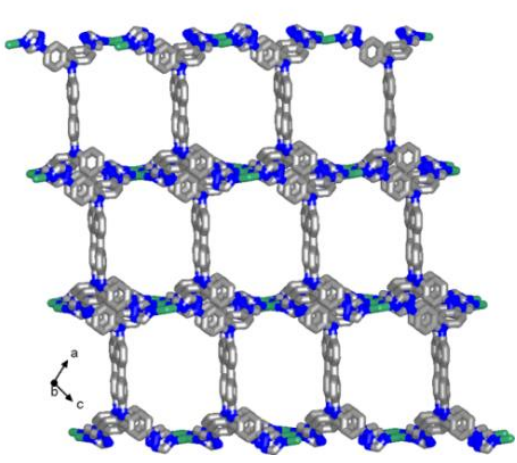

(d)

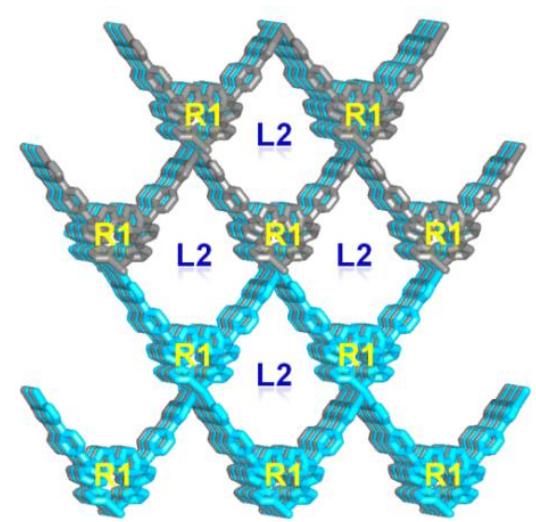

Figure S3. (a) and (b) The single 3D network constructed by Cd(II) ions and TTPBDA ligands on the $b c$ or $a c$ planes in 4. (c) and (d) Two sets of interpenetrating 3D net in 4.

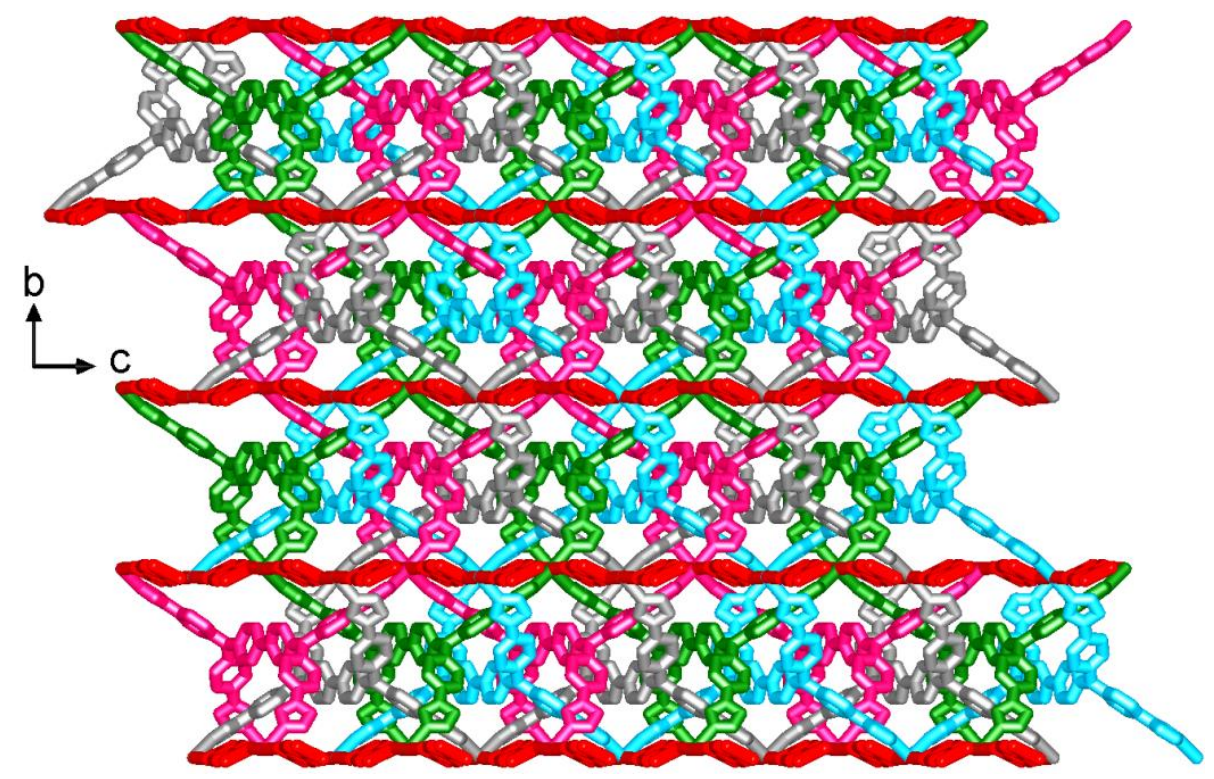

Figure S4. The self-penetrating network of 4 


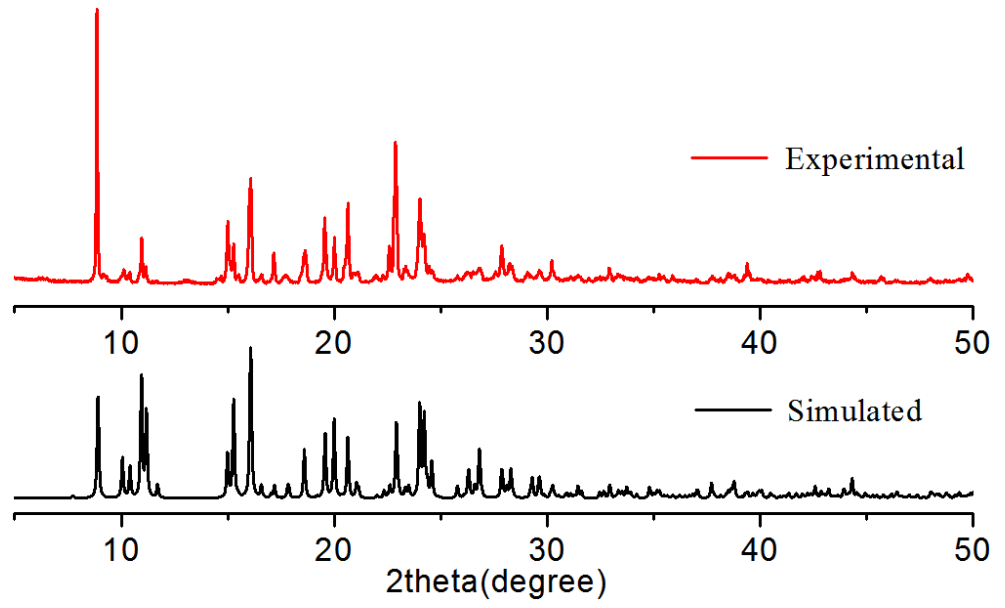

Figure S5. Powder X-ray diffraction patterns of complex 1

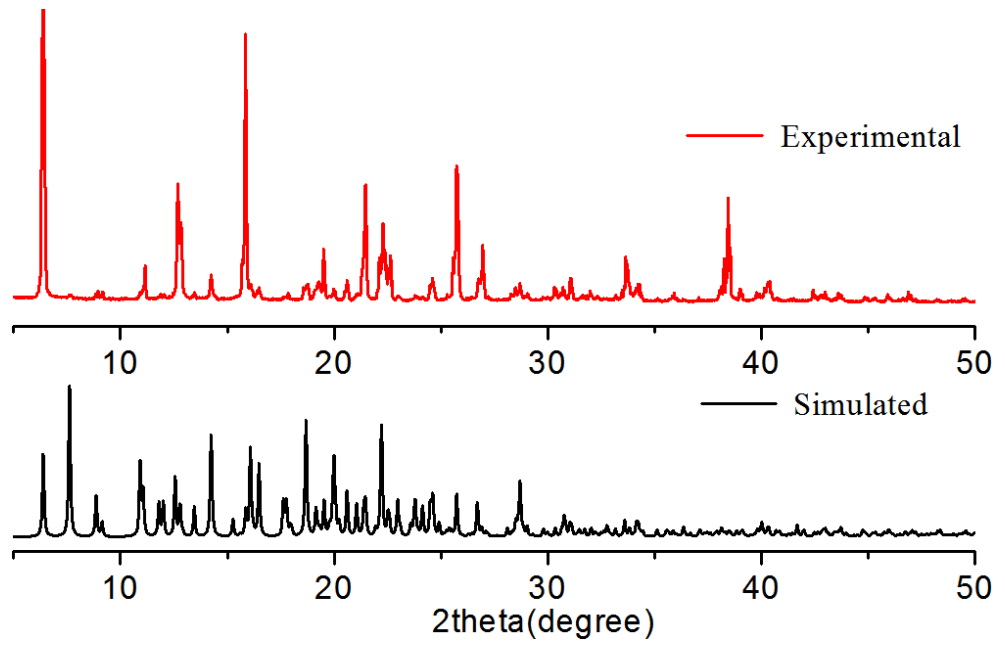

Figure S6. Powder X-ray diffraction patterns of complex 2
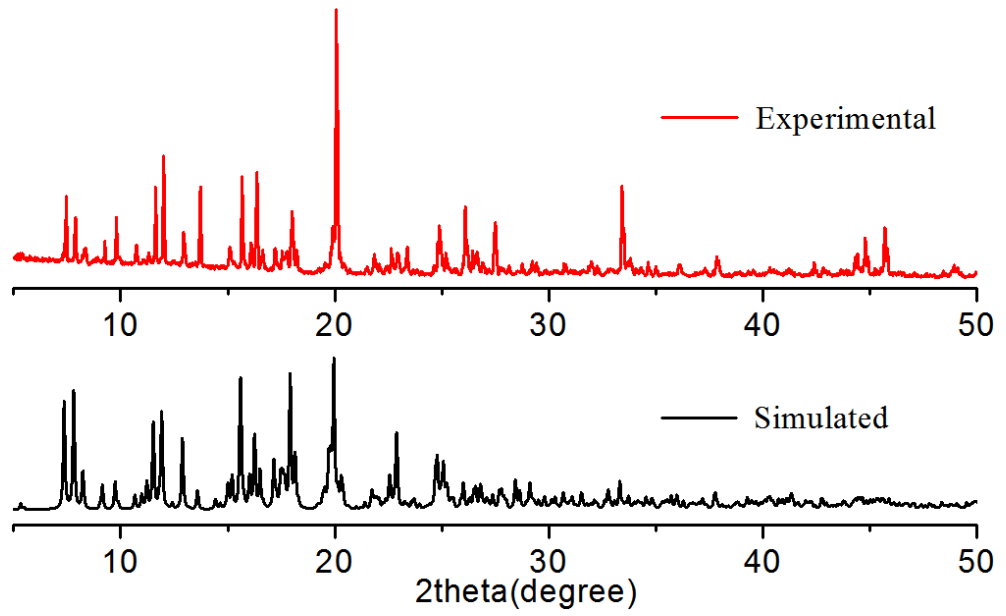

Figure S7. Powder X-ray diffraction patterns of complex 3 


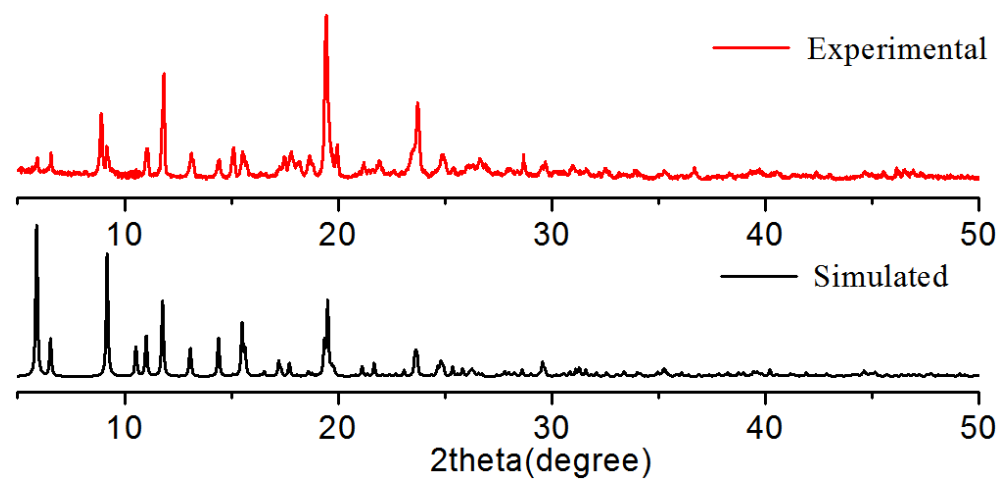

Figure S8. Powder X-ray diffraction patterns of complex 4

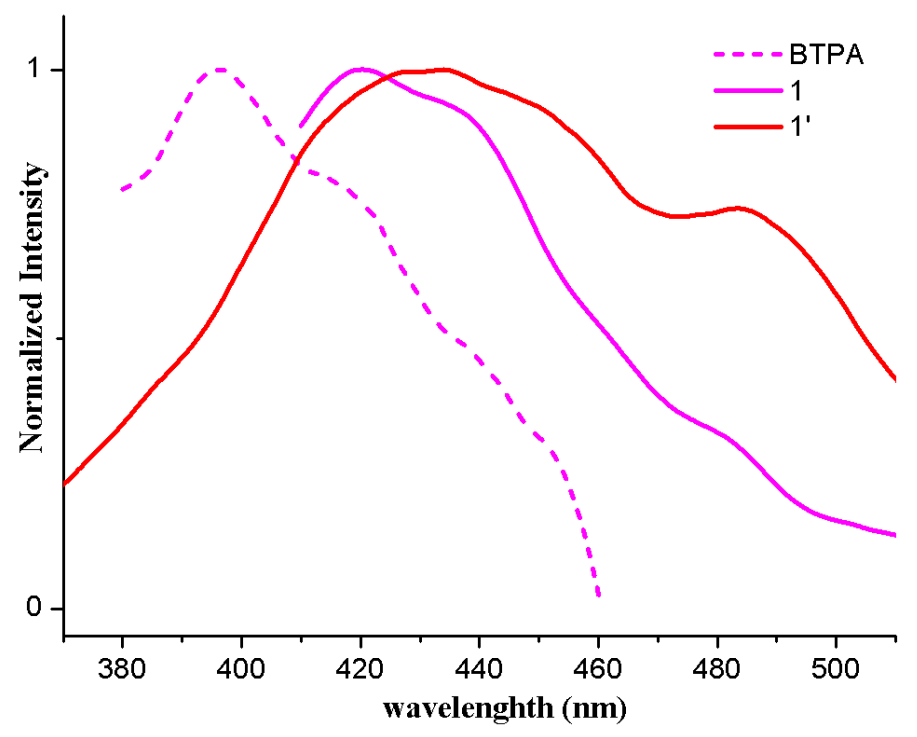

Figure S9. Emission spectra of BTPA, 1 and $\mathbf{1}^{\prime}$

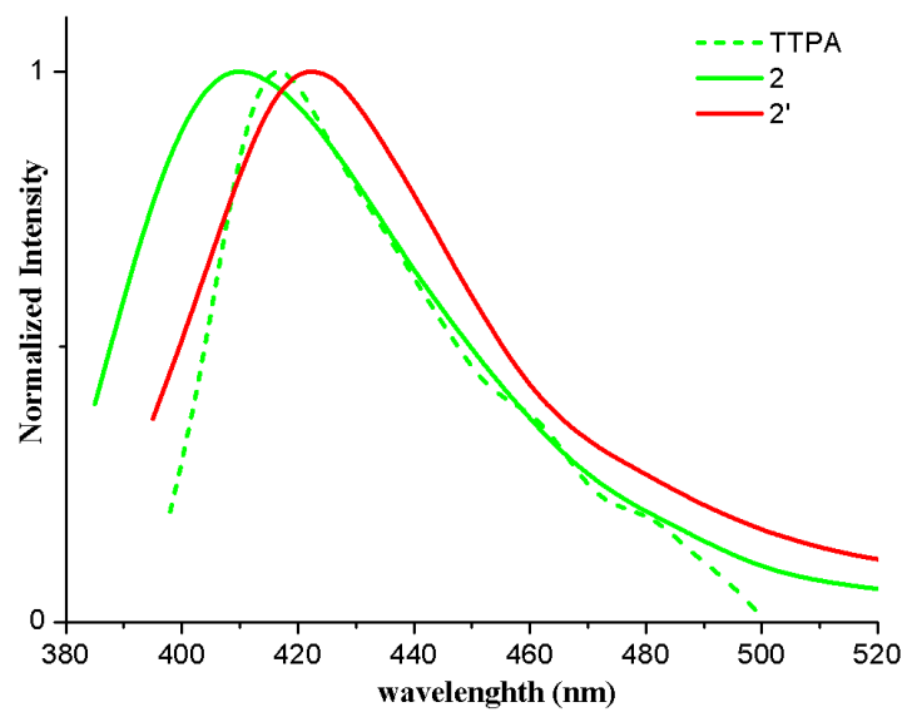

Figure S10. Emission spectra of TTPA, 2 and $\mathbf{2}^{\prime}$ 


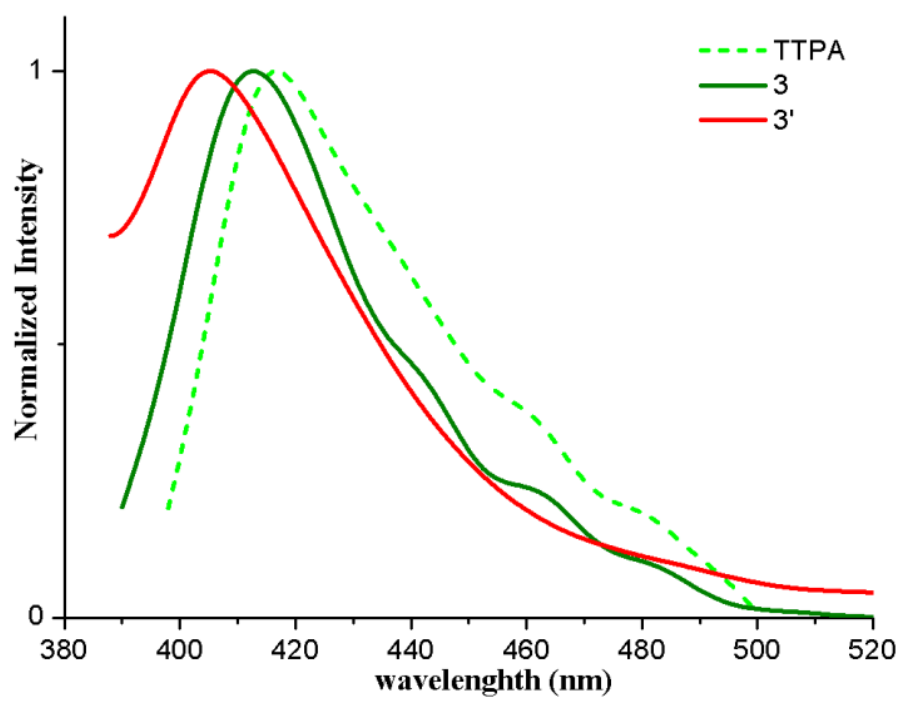

Figure S11. Emission spectra of TTPA, 3 and $\mathbf{3}^{\prime}$

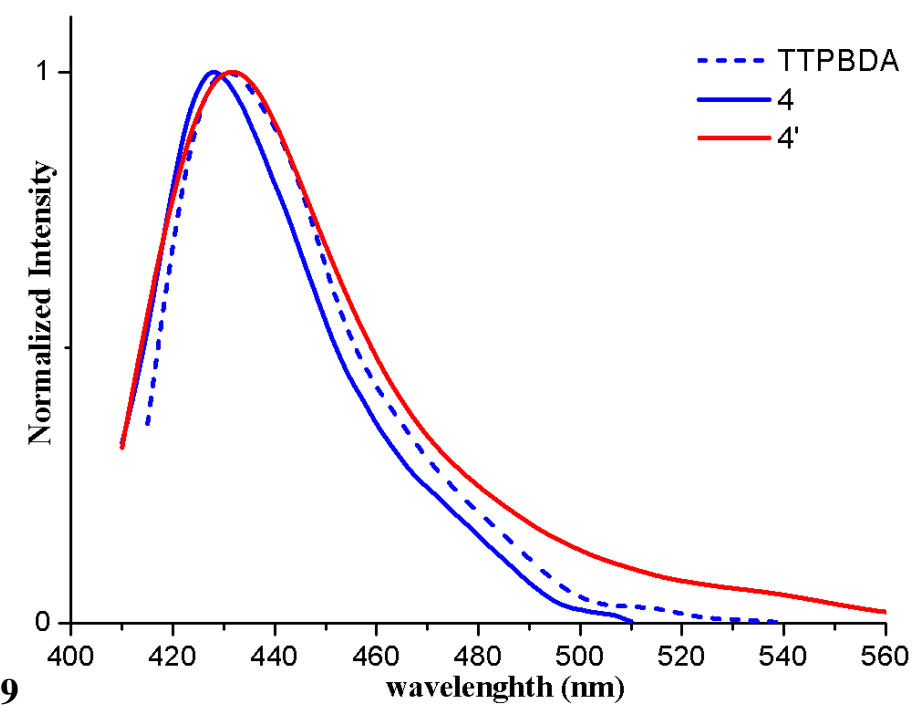

Figure S12. Emission spectra of TTPBDA, 4 and $\mathbf{4}^{\prime}$

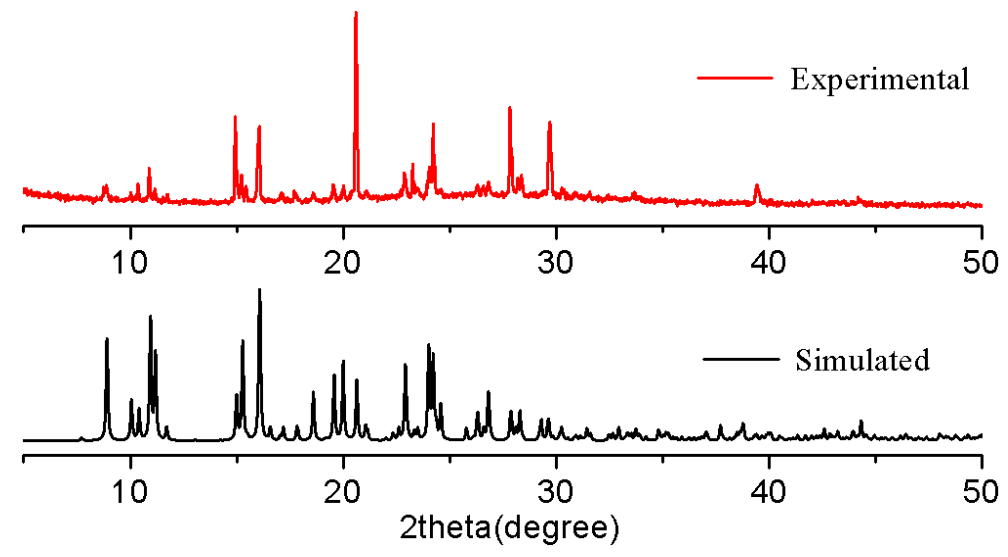

Figure S13. Powder X-ray diffraction patterns of complex 1' 


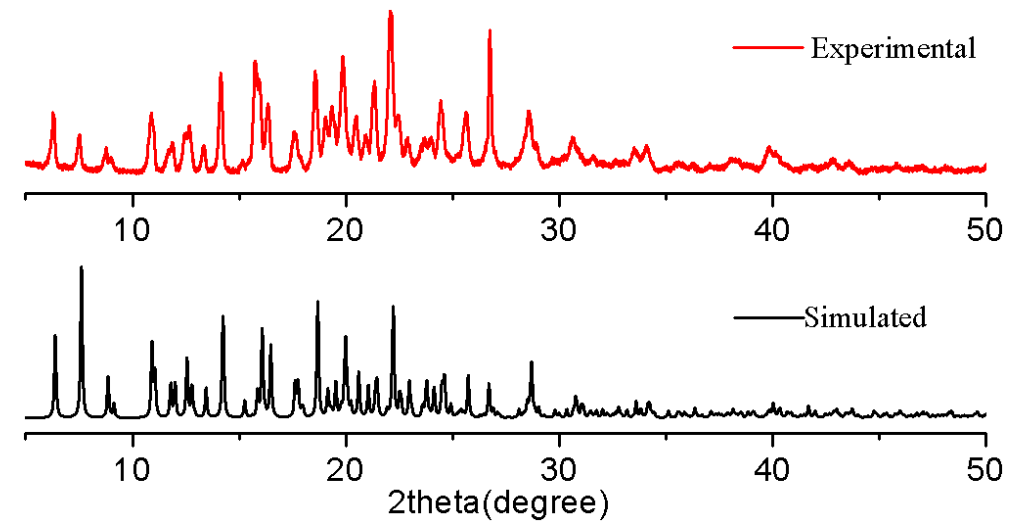

Figure S14. Powder X-ray diffraction patterns of complex 2'
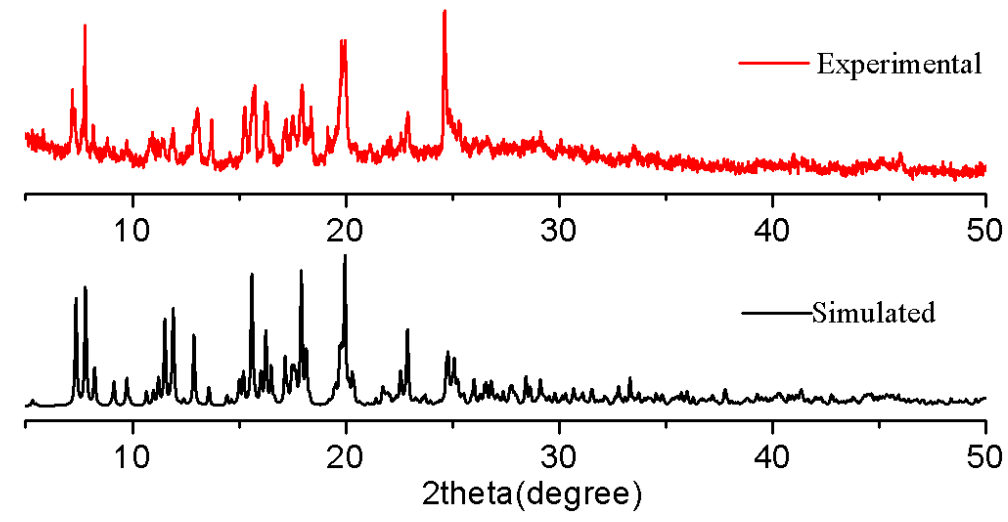

Figure S15. Powder X-ray diffraction patterns of complex $\mathbf{3}^{\prime}$
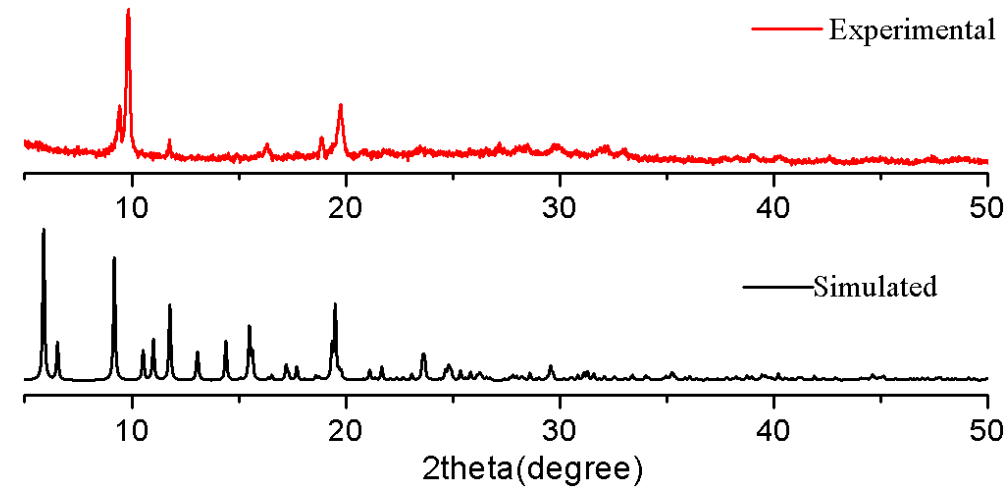

Figure S16. Powder X-ray diffraction patterns of complex $\mathbf{4}^{\prime}$ 


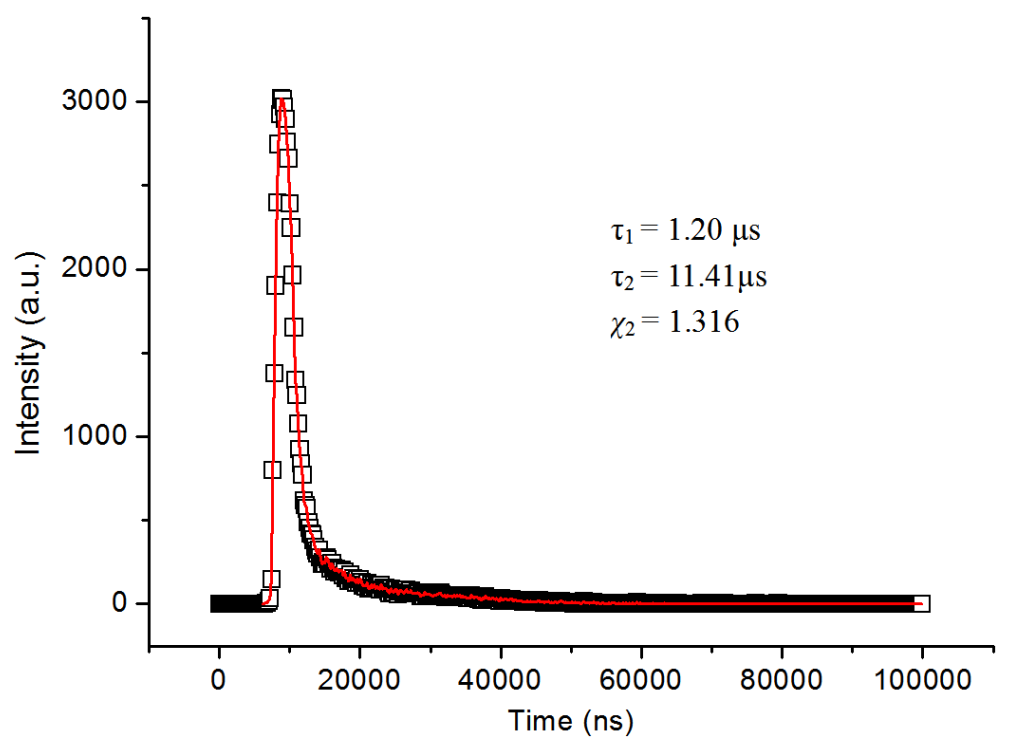

Figure S17. The fitted decay curve monitored at $420 \mathrm{~nm}$ for complex 1 in the solid state at room temperature. The sample was excited at $397 \mathrm{~nm}$. Blank open circles: experimental data; Red solid line: fitted by Fit $=\mathrm{A}+\mathrm{B}_{1} \times \exp \left(-\mathrm{t} / \tau_{1}\right)+\mathrm{B}_{2} \times \exp \left(-\mathrm{t} / \tau_{2}\right)$.

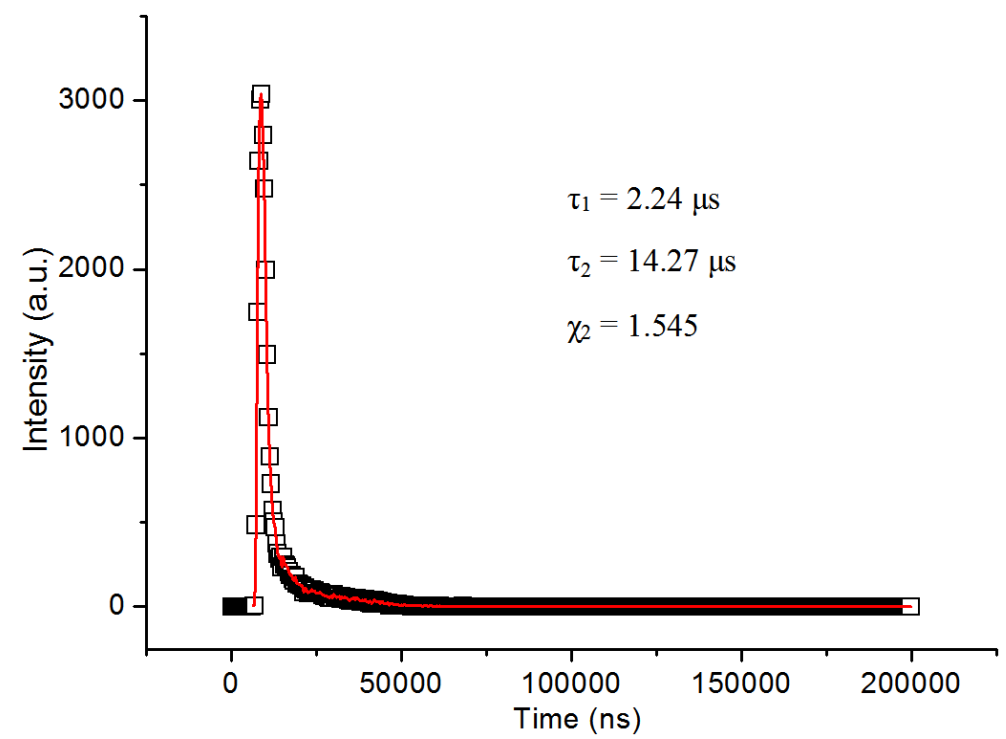

Figure S18. The fitted decay curve monitored at $407 \mathrm{~nm}$ for complex $\mathbf{2}$ in the solid state at room temperature. The sample was excited at $369 \mathrm{~nm}$. Blank open circles: experimental data; Red solid line: fitted by Fit $=\mathrm{A}+\mathrm{B}_{1} \times \exp \left(-\mathrm{t} / \tau_{1}\right)+\mathrm{B}_{2} \times \exp \left(-\mathrm{t} / \tau_{2}\right)$. 


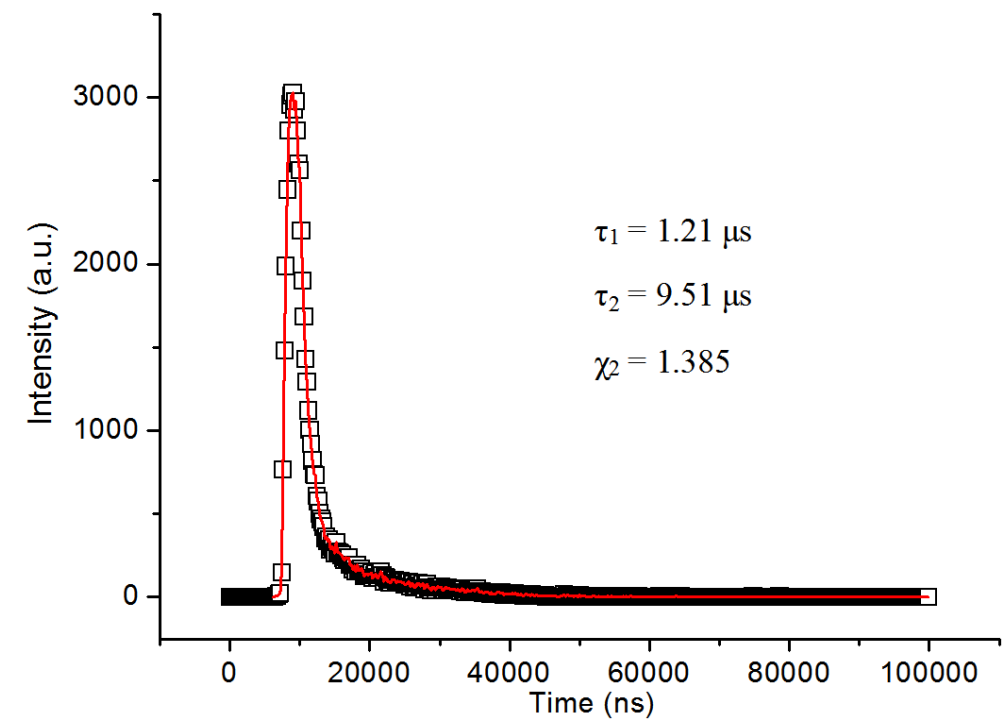

Figure S19. The fitted decay curve monitored at $413 \mathrm{~nm}$ for complex 3 in the solid state at room temperature. The sample was excited at $380 \mathrm{~nm}$. Blank open circles: experimental data; Red solid line: fitted by Fit $=\mathrm{A}+\mathrm{B}_{1} \times \exp \left(-\mathrm{t} / \tau_{1}\right)+\mathrm{B}_{2} \times \exp \left(-\mathrm{t} / \tau_{2}\right)$.

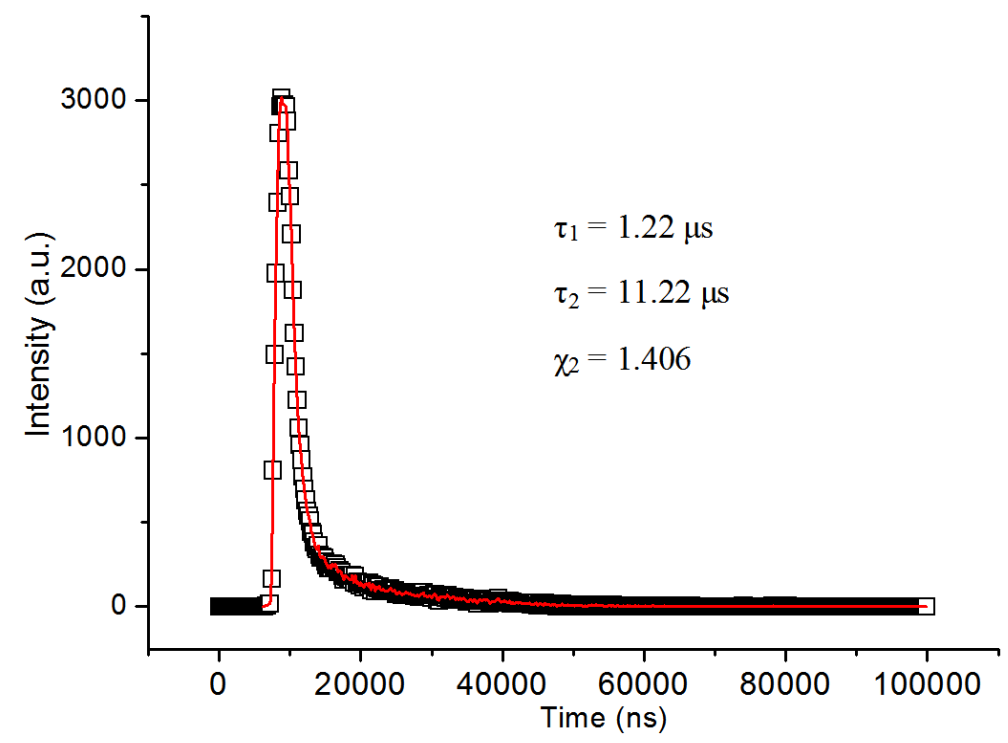

Figure S20. The fitted decay curve monitored at $428 \mathrm{~nm}$ for complex $\mathbf{4}$ in the solid state at room temperature. The sample was excited at $397 \mathrm{~nm}$. Blank open circles: experimental data; Red solid line: fitted by Fit $=\mathrm{A}+\mathrm{B}_{1} \times \exp \left(-\mathrm{t} / \tau_{1}\right)+\mathrm{B}_{2} \times \exp \left(-\mathrm{t} / \tau_{2}\right)$.

\section{Materials and methods}

All chemicals and solvents except BTPA, TTPA ${ }^{\mathrm{S} 1}$ and TTPBDA ligands were of reagent-grade quality from commercial sources and were used without further 
purification. The above ligands were prepared by the copper-catalyzed carbon-nitrogen bond cross-coupling reaction using Ullmann condensation methods based on previous literature with proper modifications. ${ }^{\text {S2 }}$ NMR spectra were recorded on a Bruker NMR 500 DRX spectrometer. The coupling constants were reported in hertz. Electrospray ionization mass spectra (ESI-MS) were recorded on a FinniganMATSSQ 710 mass spectrometer in the scan range 120-1000 amu. The IR absorption spectra of these complexes were recorded in the range of $400-4000 \mathrm{~cm}^{-1}$ by means of a Nicolet (Impact 410) spectrometer with $\mathrm{KBr}$ pellets. Element analyses (C, H, N) were carried out on a Perkin-Elmer model 240C analyzer. PXRD measurements were performed on a Bruker D8 Advance X-ray diffractometer using $\mathrm{Cu}-\mathrm{K} \alpha$ radiation $(0.15418 \mathrm{~nm})$, in which the X-ray tube was operated at $40 \mathrm{kV}$ and $30 \mathrm{~mA}$. Thermogravimetric analysis was performed on a Perkin Elmer thermogravimetric analyzer from room temperature to $700{ }^{\circ} \mathrm{C}$ with a heating rate of $20 \mathrm{~K} \cdot \mathrm{min}^{-1}$ under $\mathrm{N}_{2}$ atmosphere. Photoluminescence spectra for the solid samples were recorded with a PerkinElmer FS_LS55 fluorescence spectrophotometer at room temperature. The decay lifetimes were measured with an Edinburgh Instruments FLS920P fluorescence spectrometer in the solid state at room temperature.

\section{Synthesis of bis(4-(1H-1,2,4-triazol-1-yl)phenyl)amine (BTPA)}

A mixture of bis(4-bromophenyl)amine ${ }^{\mathrm{S} 3}$ (20.0 mmol, $\left.6.54 \mathrm{~g}\right)$, 1,2,4-triazole (200.0 mmol, $13.80 \mathrm{~g}), \mathrm{CuI}(2 \mathrm{mmol}, 0.38 \mathrm{~g}), \mathrm{CuSO}_{4}$ (1 mmol, $\left.0.16 \mathrm{~g}\right), 18$-crown-6 (2.0 mmol, $0.53 \mathrm{~g}$ ), and $\mathrm{K}_{2} \mathrm{CO}_{3}(200.0 \mathrm{mmol}, 27.6 \mathrm{~g})$ was suspended in $50 \mathrm{~mL}$ of DMF. The mixture in a $250-\mathrm{mL}$ two-necked round-bottom flask under $\mathrm{N}_{2}$ was refluxed at $160{ }^{\circ} \mathrm{C}$ for 3 days and then cooled to room temperature. Solvent was removed by distillation under a vacuum, and the reaction mixture was added into $200 \mathrm{~mL} \mathrm{H}_{2} \mathrm{O}$. The deposit was filtered and washed with water and dried in vacuum to obtain light blue powder. Then the crude product was separated by column chromatography (petroleum ether/ethyl acetate $=1: 1$ ) to afford white crystalline powder (yield: $\sim 74 \%$, $4.51 \mathrm{~g}$, based on bis(4-bromophenyl)amine). ${ }^{1} \mathrm{H}$ NMR (500 MHz, DMSO-d 6 ), $\delta$ (ppm): $9.15(\mathrm{~s}, 2 \mathrm{H}), 8.57(\mathrm{~s}, 1 \mathrm{H}), 8.19(\mathrm{~s}, 2 \mathrm{H}), 7.73(\mathrm{~d}, J=9.0 \mathrm{~Hz}, 4 \mathrm{H}), 7.26(\mathrm{~d}, J=9.0 \mathrm{~Hz}$, 
4H). Anal. Calcd for $\mathrm{C}_{16} \mathrm{H}_{13} \mathrm{~N}_{7}: \mathrm{C}, 63.36 ; \mathrm{H}, 4.32 ; \mathrm{N}, 32.32$. Found: $\mathrm{C}, 63.31 ; \mathrm{H}, 4.27$; $\mathrm{N}, 31.38$. IR $\left(\mathrm{KBr}, \mathrm{cm}^{-1}\right): 3264 \mathrm{~m}, 3200 \mathrm{~m}, 3112 \mathrm{~m}, 1606 \mathrm{~m}, 1512 \mathrm{~s}, 1318 \mathrm{~m}, 1275 \mathrm{~m}$, 1208m, 1173w, 1140m, 1049m, 954w, 859w, 817m, 677m, 643m, 524m MS (ESI), $\mathrm{m} / \mathrm{z}(\%): 304.25$ for $\mathrm{C}_{16} \mathrm{H}_{14} \mathrm{~N}_{7}^{+}$. (Figure S21-S23)

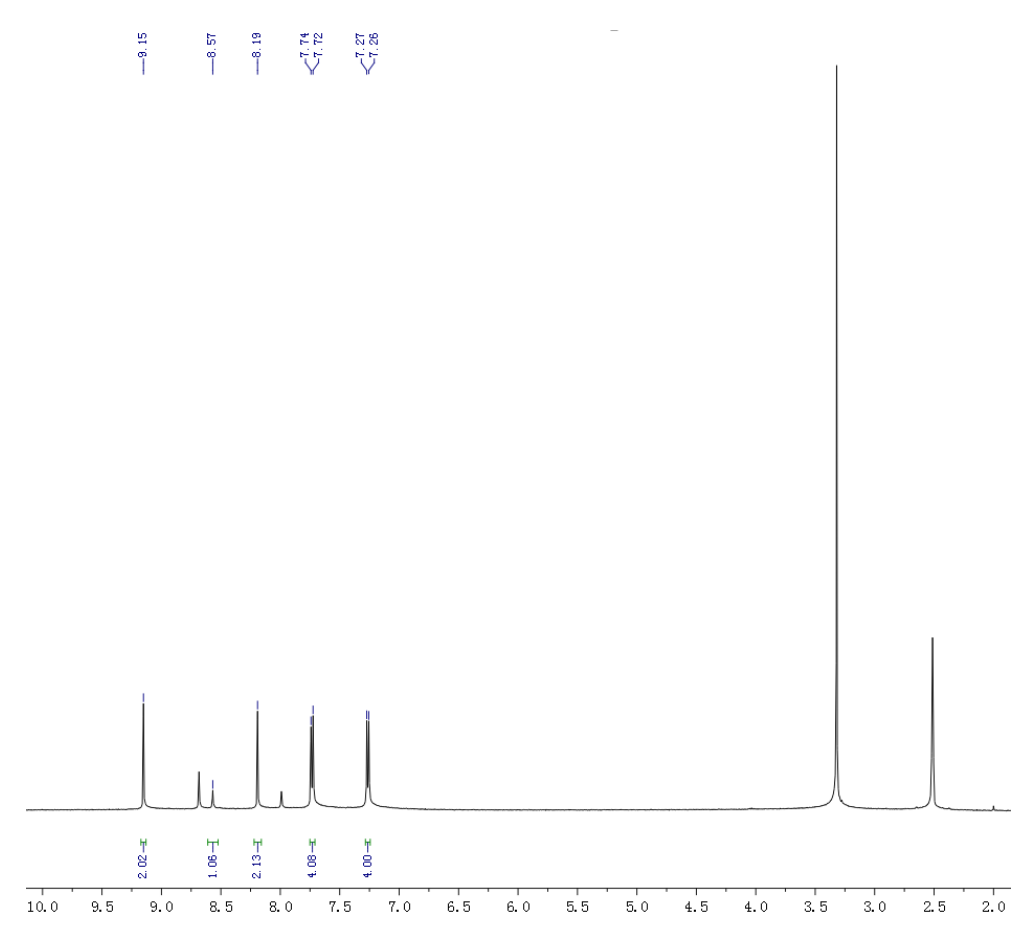

Figure S21. The ${ }^{1} \mathrm{H}$ NMR spectra of BTPA

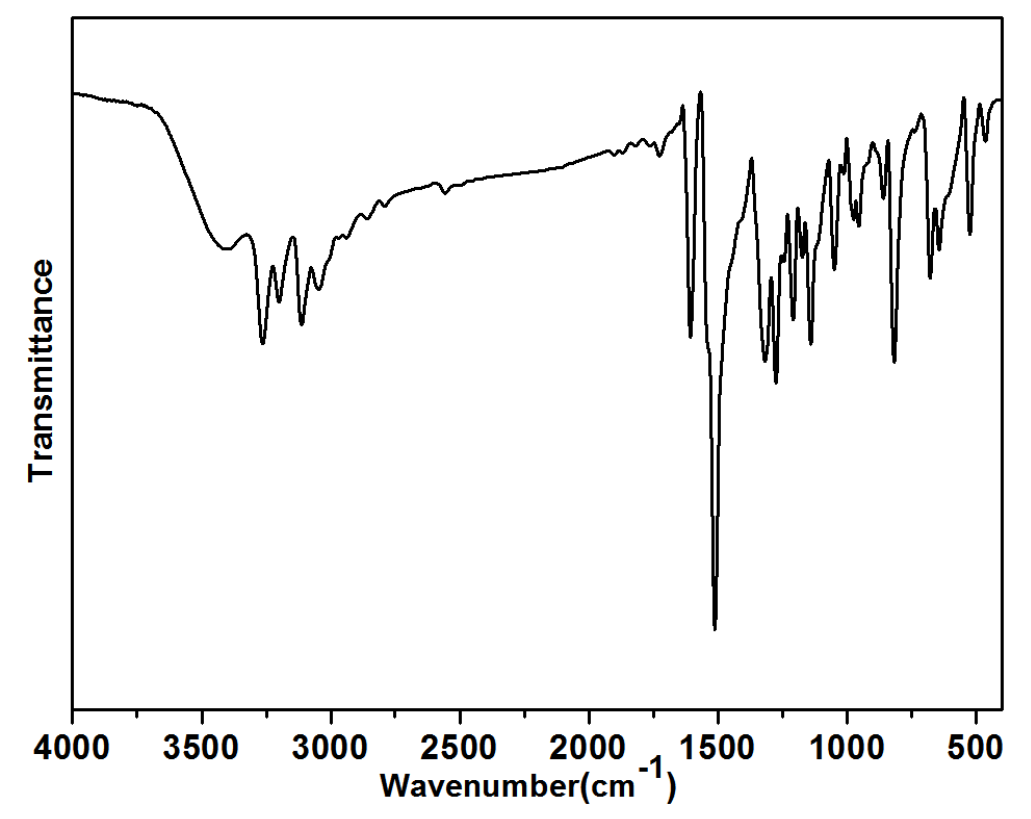

Figure S22. FT-IR spectroscopy of BTPA 


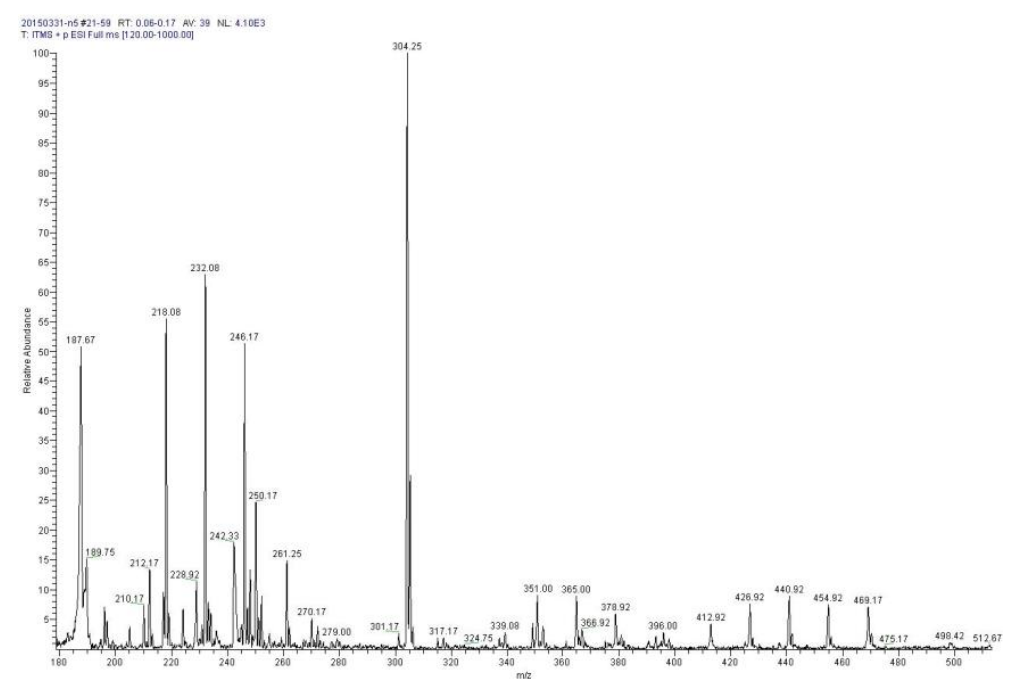

Figure S23. The EI-MS scheme of BTPA

Synthesis of $N^{4}, N^{4}, N^{4}, N^{4}$-tetrakis(4-(1H-1,2,4-triazol-1-yl)phenyl)-(1,1'-biphenyl )-4,4'-diamine (TTBPDA)

A mixture of $N^{4}, N^{4}, N^{4}, N^{41}$-tetrakis(4-bromophenyl)-(1,1'-biphenyl)-4,4'-diamine ${ }^{\mathrm{S} 4}$ (3.0 mmol, $2.41 \mathrm{~g})$, 1,2,4-triazole (45.0 mmol, $3.11 \mathrm{~g})$, CuI (0.15 mmol, $0.029 \mathrm{~g})$, 18-crown-6 (1.5 mmol, $0.4 \mathrm{~g}$ ), and $\mathrm{K}_{2} \mathrm{CO}_{3}$ (45.0 mmol, $6.22 \mathrm{~g}$ ) was suspended in 30 $\mathrm{mL}$ of DMF. The mixture in a 100-mL two-necked round-bottom flask under $\mathrm{N}_{2}$ was refluxed at $160{ }^{\circ} \mathrm{C}$ for 3 days and then cooled to room temperature. Solvent was removed by distillation under a vacuum, and the reaction mixture was added into 100 $\mathrm{mL} \mathrm{H}_{2} \mathrm{O}$. The deposit was filtered and washed with water and dried in vacuum to obtain light blue powder. Then the crude product was separated by column chromatography (ethyl acetate/methanol $=10: 1$ ) to afford white crystalline powder $\begin{array}{lllll}\text { (yield: } & \sim 55 \%, & 1.26 & \mathrm{~g}, & \text { based }\end{array}$ $N^{4}, N^{4}, N^{4 !}, N^{41}$-tetrakis(4-bromophenyl)-(1,1'-biphenyl)-4,4'-diamine). ${ }^{1} \mathrm{H}$ NMR (500 MHz, DMSO-d 6 ), $\delta(\mathrm{ppm}): 9.23(\mathrm{~s}, 4 \mathrm{H}), 8.21(\mathrm{~s}, 4 \mathrm{H}), 7.84(\mathrm{~d}, J=15.0 \mathrm{~Hz}, 8 \mathrm{H}), 7.68$ $(\mathrm{d}, J=15.0 \mathrm{~Hz}, 4 \mathrm{H}), 7.25(\mathrm{~d}, J=15.0 \mathrm{~Hz}, 8 \mathrm{H}), 7.18(\mathrm{~d}, J=15.0 \mathrm{~Hz}, 4 \mathrm{H})$. Anal. Calcd for $\mathrm{C}_{44} \mathrm{H}_{32} \mathrm{~N}_{14}$ : C, 69.83; H, 4.26; N, 25.91. Found: C, 69.79; H, 4.30; N, 25.88. IR $\left(\mathrm{KBr}, \mathrm{cm}^{-1}\right): 3400 \mathrm{~s}, 3125 \mathrm{~s}, 1684 \mathrm{~m}, 1604 \mathrm{~m}, 1516 \mathrm{~s}, 1491 \mathrm{~s}, 1400 \mathrm{~s}, 1325 \mathrm{~m}, 1274 \mathrm{~s}$, 1213m, 1178w, 1007w, 979m, 826m, 722w, 674m, 534w. MS (ESI), m/z (\%): 757.50. 
for $\mathrm{C}_{44} \mathrm{H}_{33} \mathrm{~N}_{14}{ }^{+}$(Figure S24-S26).

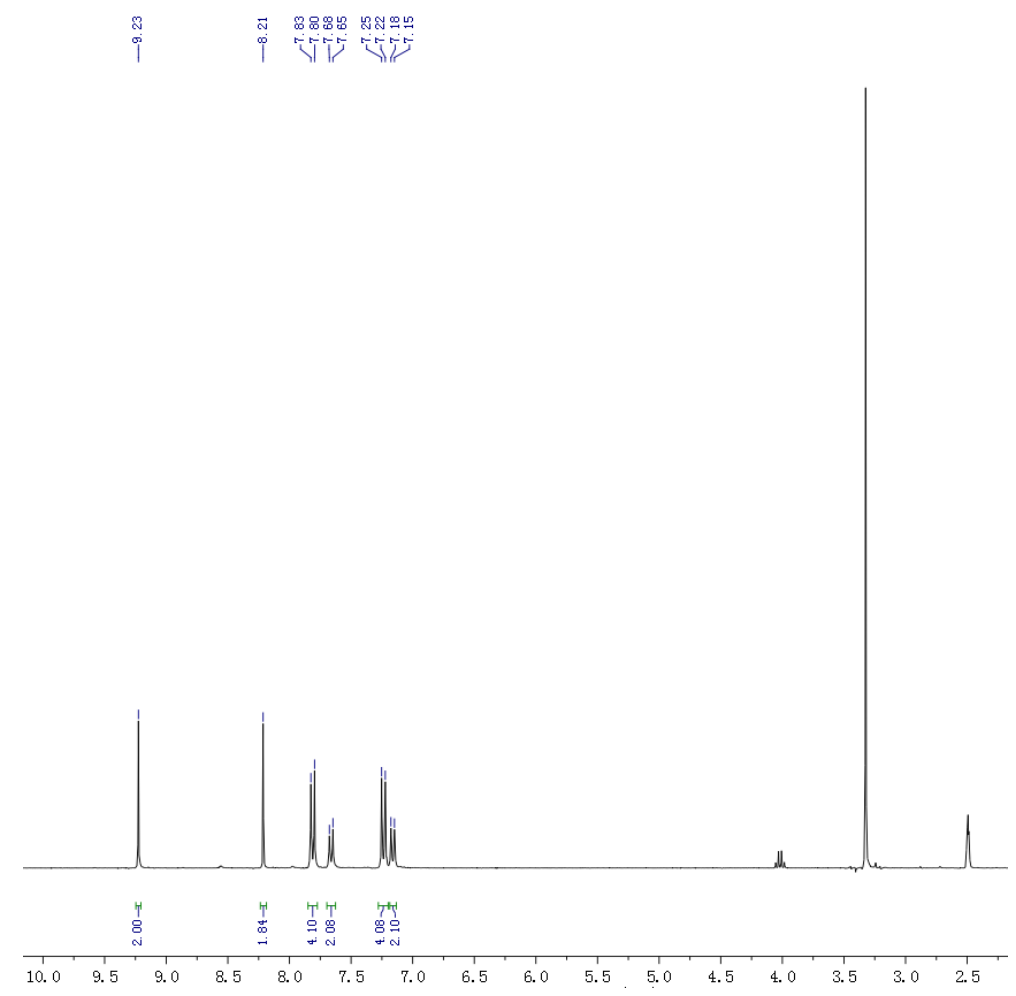

Figure S24. The ${ }^{1} \mathrm{H}$ NMR spectra of TTBPDA

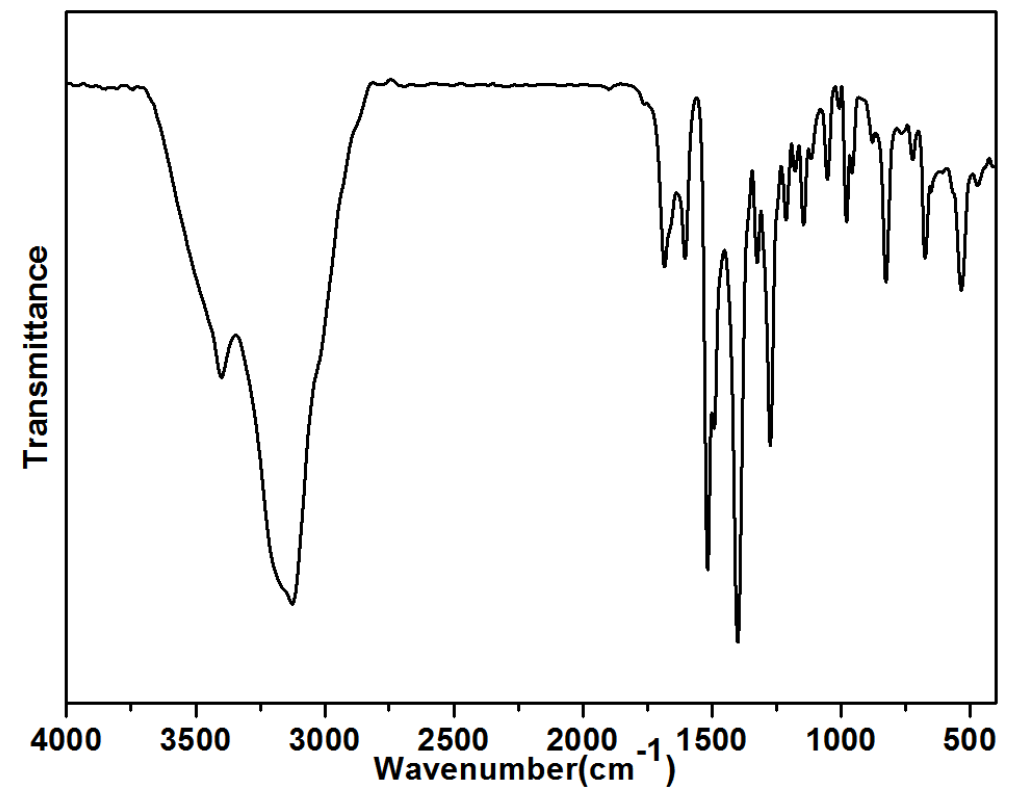

Figure S25. FT-IR spectroscopy of TTBPDA 


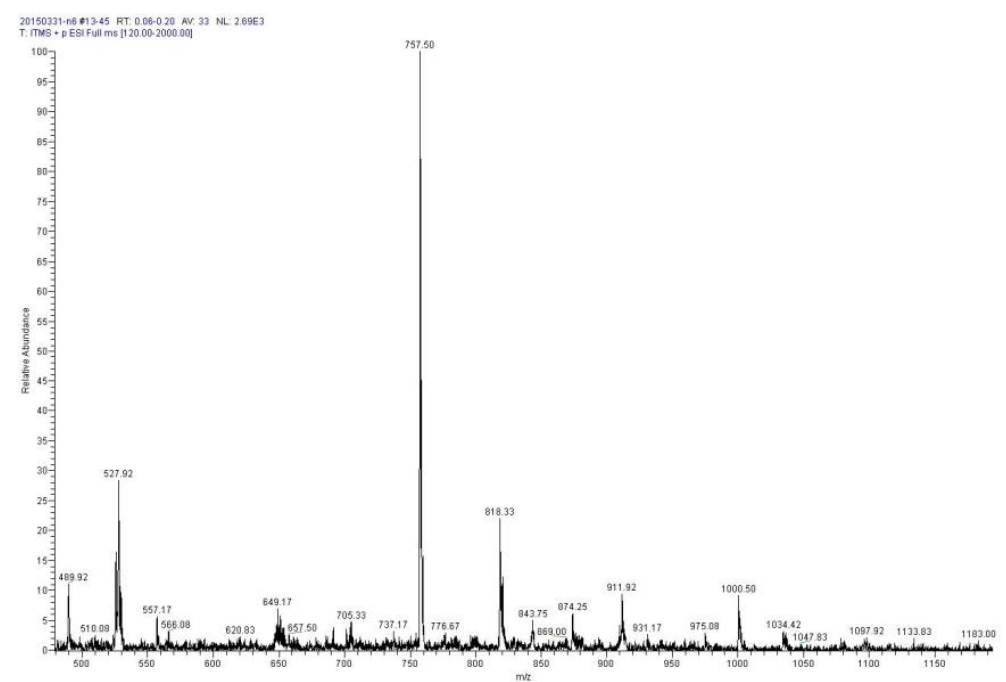

Figure S26 The EI-MS scheme of TTBPDA

\section{Crystal Structure Determination}

Single crystals of 1-4 were prepared by the methods described in the synthetic procedure. Their crystal structures were determined by single-crystal X-ray analyses. Data collections were performed using a Bruker Smart Apex CCD diffractometer with Mo- $K \alpha$ radiation with an $\varphi-\omega$ mode $(\lambda=0.71073 \AA)$. The structures were solved with direct methods using the SHELXTL program $^{\mathrm{S} 5}$ and refined anisotropically with SHELXTL using full-matrix least-squares procedures. In the crystal X-ray structure analysis, some residual electron densities of complexes $\mathbf{1}$ and $\mathbf{4}$ were observed within the pores, and assignment to specific molecules cannot be made. They were removed by the SQUEEZE routine in PLATON.

\section{Preparation of the Complexes 1-4}

Synthesis of $\left\{[\mathbf{Z n}(\mathbf{B T P A})(\mathrm{TPA})] \cdot \mathrm{H}_{2} \mathrm{O}\right\}_{n}$ (1). A mixture of $\mathrm{DMF} / \mathrm{CH}_{3} \mathrm{CN} / \mathrm{H}_{2} \mathrm{O}(2 / 1 / 3$ $\mathrm{ml})$ containing BTPA (4.55 mg, $0.015 \mathrm{mmol}), \mathrm{H}_{2}$ TPA $(8.30 \mathrm{mg}, 0.05 \mathrm{mmol})$, and $\mathrm{Zn}\left(\mathrm{NO}_{3}\right)_{2} \cdot 6 \mathrm{H}_{2} \mathrm{O}(29.7 \mathrm{mg}, 0.1 \mathrm{mmol})$ was placed in a Teflon vessel $(12 \mathrm{~mL})$ under autogenous pressure and heated at $85{ }^{\circ} \mathrm{C}$ for 72 hours and then cooled to room temperature over $24 \mathrm{~h}$. Colorless block-shaped crystals of 1 were obtained, dried in air and collected in 51\% yield based on the BTPA ligand. Elemental analysis calcd. for $\mathrm{C}_{24} \mathrm{H}_{19} \mathrm{~N}_{7} \mathrm{O}_{5} \mathrm{Zn}(\%)$ : C, 52.33; H, 3.48; N, 17.80. Found: C, 52.25; H, 3.39; N, 17.88. 
IR $\left(\mathrm{KBr}, \mathrm{cm}^{-1}\right): 3412 \mathrm{~s}, 3283 \mathrm{~s}, 3134 \mathrm{~m}, 1606 \mathrm{vs}, 1535 \mathrm{~s}, 1356 \mathrm{~s}, 1291 \mathrm{~m}, 1215 \mathrm{w}, 1150 \mathrm{~m}$, 1051w, 975w, 824m, 744m, 667w, 639w, 574w (Figure S27).

Synthesis of $\left\{[\mathbf{Z n}(\mathbf{T T P A})(\mathbf{T P A})] \cdot \mathrm{H}_{2} \mathrm{O}\right\}_{n}$ (2). A mixture of $\mathrm{DMF} / \mathrm{H}_{2} \mathrm{O}(2 / 6 \mathrm{ml})$ and 2 drops $\mathrm{CH}_{3} \mathrm{CN}$ containing TTPA (4.46 mg, $0.01 \mathrm{mmol}$ ), $\mathrm{H}_{2}$ TPA (16.60 mg, $0.1 \mathrm{mmol}$ ), and $\mathrm{Zn}\left(\mathrm{NO}_{3}\right)_{2} \cdot 6 \mathrm{H}_{2} \mathrm{O}(29.70 \mathrm{mg}, 0.1 \mathrm{mmol})$ was placed in a Teflon vessel $(12 \mathrm{~mL})$ under autogenous pressure and heated at $95{ }^{\circ} \mathrm{C}$ for 72 hours and then cooled to room temperature over $24 \mathrm{~h}$. Colorless block-shaped crystals of $\mathbf{2}$ were obtained, dried in air and collected in 58\% yield based on the TTPA ligand. Elemental analysis calcd. for $\mathrm{C}_{32} \mathrm{H}_{24} \mathrm{~N}_{10} \mathrm{O}_{5} \mathrm{Zn}(\%)$ : C, 55.38; H, 3.49; N, 20.18. Found: C, 55.32; H, 3.43; N, 20.28. IR $\left(\mathrm{KBr}, \mathrm{cm}^{-1}\right): 3409 \mathrm{~m}, 3136 \mathrm{~m}, 3091 \mathrm{~m}, 1683 \mathrm{w}, 1595 \mathrm{vs}, 1513 \mathrm{vs}, 1354 \mathrm{vs}, 1279 \mathrm{vs}$, $1232 \mathrm{~m}, 1177 \mathrm{~m}, 1144 \mathrm{~s}, 1051 \mathrm{~m}, 1003 \mathrm{~s}, 977 \mathrm{~s}, 882 \mathrm{w}, 822 \mathrm{~m}, 750 \mathrm{~s}, 674 \mathrm{~m}, 653 \mathrm{~m}, 591 \mathrm{~m}$, 539s (Figure S28).

Synthesis of $\left\{\left[\mathrm{Cd}_{2}(\mathrm{TTPA})_{2}(\mathrm{TPA})_{2}(\mathrm{DMF})\right] \cdot \mathbf{2 H}_{2} \mathrm{O}\right\}_{\mathbf{n}}(\mathbf{3})$. A mixture of $\mathrm{DMF} / \mathrm{H}_{2} \mathrm{O}(2 / 4$ $\mathrm{ml}$ ) and 1 drop $\mathrm{CH}_{3} \mathrm{CN}$ containing TTPA (4.46 mg, $0.01 \mathrm{mmol}$ ), $\mathrm{H}_{2} \mathrm{TPA}$ (8.30 mg, $0.05 \mathrm{mmol})$, and $\mathrm{Cd}\left(\mathrm{NO}_{3}\right)_{2} \cdot 4 \mathrm{H}_{2} \mathrm{O}(15.4 \mathrm{mg}, 0.05 \mathrm{mmol})$ was placed in a Teflon vessel $(12 \mathrm{~mL})$ under autogenous pressure and heated at $105^{\circ} \mathrm{C}$ for 72 hours and then cooled to room temperature over 24 h. Colorless rod-shaped crystals of $\mathbf{3}$ were obtained, dried in air and collected in $62 \%$ yield based on the TTPA ligand. Elemental analysis calcd. for $\mathrm{C}_{67} \mathrm{H}_{55} \mathrm{Cd}_{2} \mathrm{~N}_{21} \mathrm{O}_{11}(\%)$ : C, 51.75; H, 3.56; N, 18.91. Found: C, 51.70; $\mathrm{H}$, 3.49; N, 19.01. IR (KBr, cm $\left.{ }^{-1}\right): 3418 \mathrm{~m}, 3068 \mathrm{~m}, 1653 \mathrm{~s}, 1571 \mathrm{vs}, 1517 \mathrm{vs}, 1442 \mathrm{~m}$, $1377 \mathrm{vs}, 1322 \mathrm{~s}, 1277 \mathrm{vs}, 1218 \mathrm{~s}, 1178 \mathrm{~m}, 1144 \mathrm{~s}, 1110 \mathrm{~m} \mathrm{1049m,1014w,} \mathrm{975s,} \mathrm{903w,}$ 833vs, 748s, 671m, 524m. (Figure S29).

Synthesis of $\left\{[\text { Cd(TTPBDA)(TPA) }]_{0.5} \cdot \text { DMF } \cdot \mathrm{H}_{2} \mathrm{O}\right\}_{n}$ (4). A mixture of DMF/ $\mathrm{H}_{2} \mathrm{O}$ (2/2 ml) containing TTPBDA (2.27 mg, $0.003 \mathrm{mmol}), \mathrm{H}_{2}$ TPA (8.30 mg, $\left.0.05 \mathrm{mmol}\right)$, and $\mathrm{Cd}\left(\mathrm{NO}_{3}\right)_{2} \cdot 4 \mathrm{H}_{2} \mathrm{O}(15.4 \mathrm{mg}, 0.05 \mathrm{mmol})$ was placed in a Teflon vessel $(12 \mathrm{~mL})$ under autogenous pressure and heated at $85{ }^{\circ} \mathrm{C}$ for 72 hours and then cooled to room temperature over $24 \mathrm{~h}$. Colorless needle-shaped crystals of 4 were obtained, dried in air and collected in 53\% yield based on the TTPBDA ligand. Elemental analysis calcd. for $\mathrm{C}_{29} \mathrm{H}_{27} \mathrm{Cd}_{0.5} \mathrm{~N}_{8} \mathrm{O}_{4}(\%)$ : C, 39.55; H, 9.96; N, 15.37. Found: C, 39.43; H, 9.89; N, 15.48. IR (KBr, cm $\left.{ }^{-1}\right): 3401 \mathrm{~s}, 3130 \mathrm{~s}, 1666 \mathrm{~m}, 1605 \mathrm{~m}, 1571 \mathrm{~m}, 1518 \mathrm{~m}, 1400 \mathrm{~s}, 1323 \mathrm{~m}$, 
1275w, 1217w, 1141w, 1051w, 974w, 826w, 749w, 670w, 540w (Figure S30).

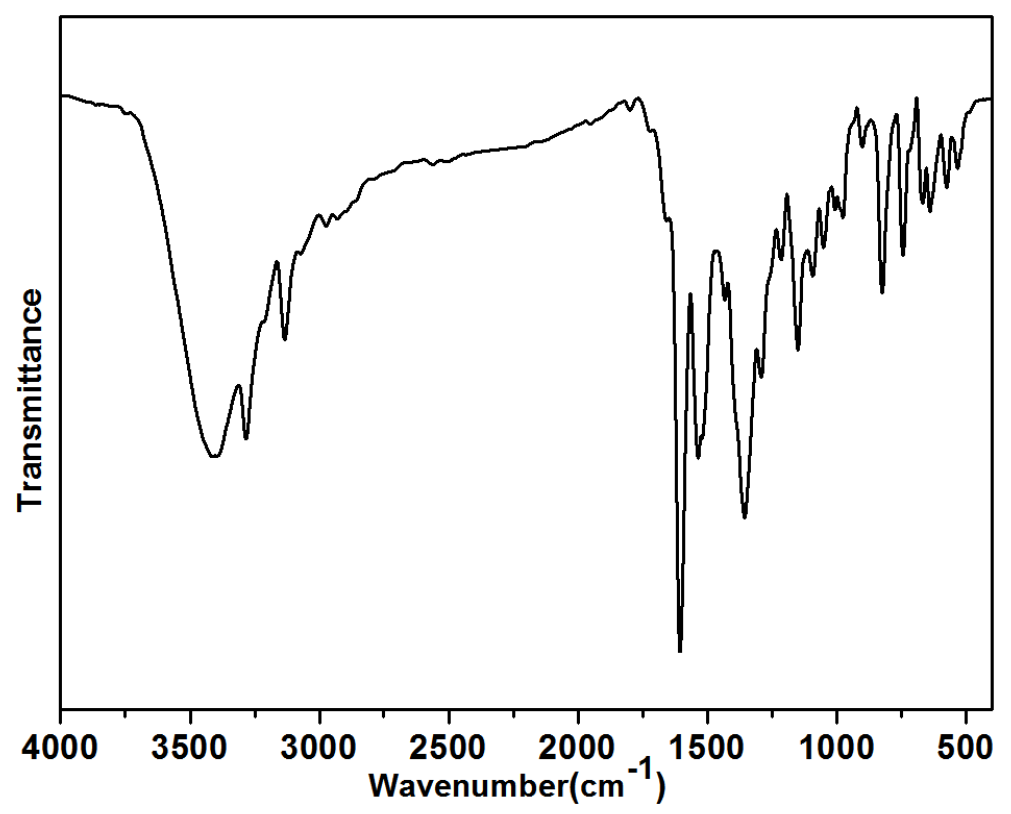

Figure S27. IR spectra of complex 1

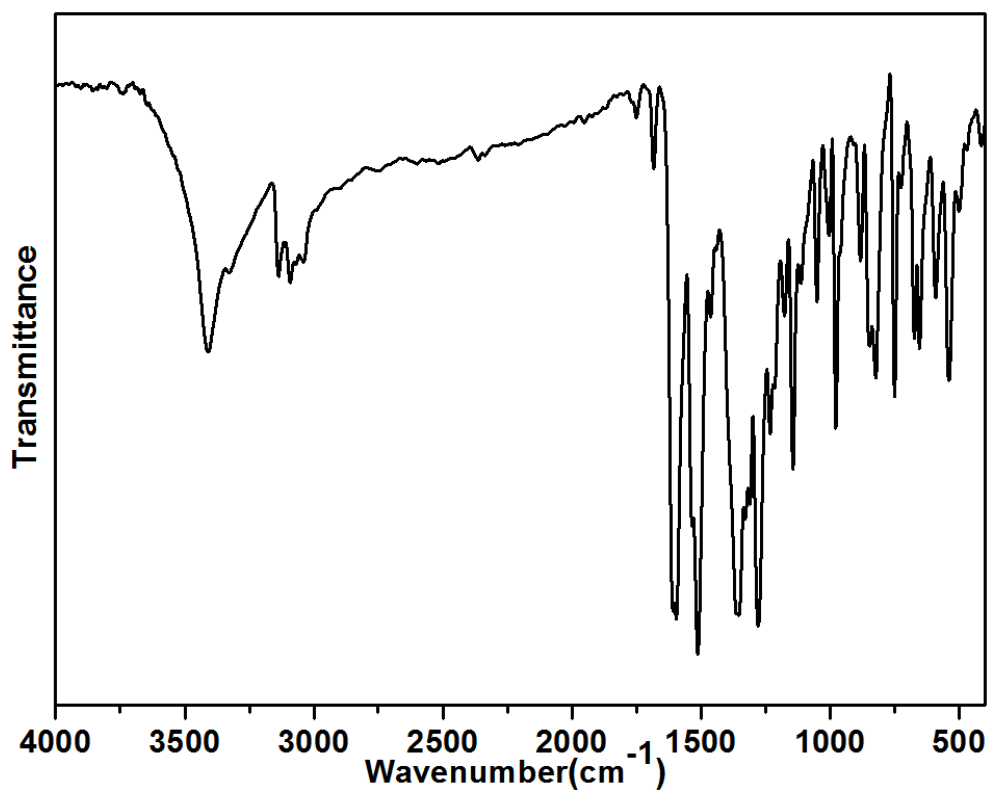

Figure S28. IR spectra of complex 2 


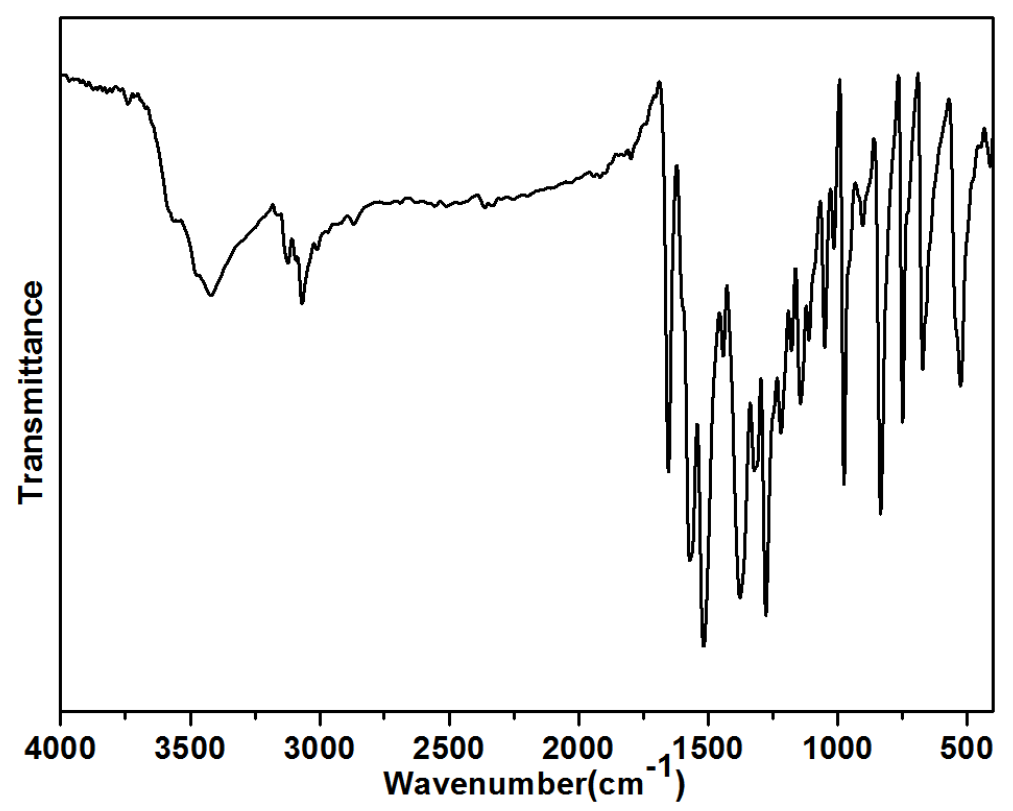

Figure S29. IR spectra of complex 3

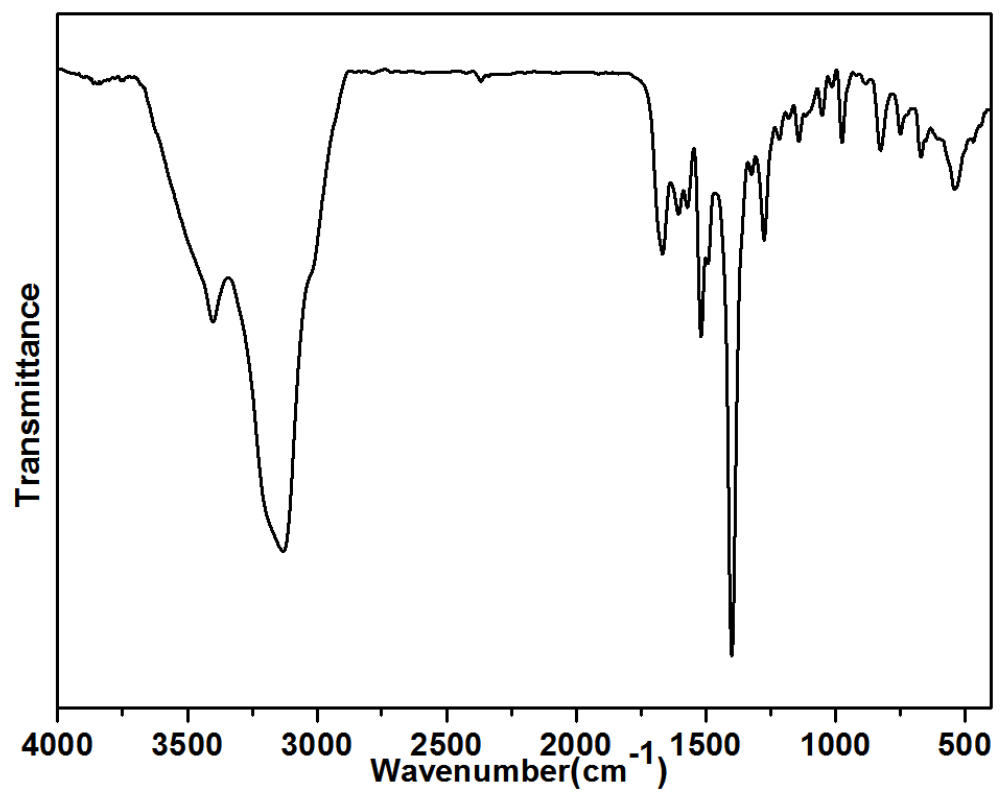

Figure S30. IR spectra of complex 4

Table S1. Selected Bond Lengths $(\AA)$ and Angles $\left({ }^{\circ}\right)$ for Complexes 1-4

\begin{tabular}{llll}
\hline \multicolumn{3}{c}{ Complex 1 } \\
\hline $\mathrm{Zn}(1)-\mathrm{O}(3)$ & $1.9548(19)$ & $\mathrm{Zn}(1)-\mathrm{N}(4) \# 1$ & $2.004(2)$ \\
$\mathrm{Zn}(1)-\mathrm{O}(1)$ & $1.9564(19)$ & $\mathrm{Zn}(1)-\mathrm{N}(1)$ & $2.028(2)$ \\
$\mathrm{O}(3)-\mathrm{Zn}(1)-\mathrm{O}(1)$ & $109.76(9)$ & $\mathrm{O}(3)-\mathrm{Zn}(1)-\mathrm{N}(1)$ & $101.61(9)$
\end{tabular}




\begin{tabular}{|c|c|c|c|}
\hline $\mathrm{O}(3)-\mathrm{Zn}(1)-\mathrm{N}(4) \# 1$ & $113.35(9)$ & $\mathrm{O}(1)-\mathrm{Zn}(1)-\mathrm{N}(1)$ & $105.38(9)$ \\
\hline $\mathrm{O}(1)-\mathrm{Zn}(1)-\mathrm{N}(4) \# 1$ & $111.54(9)$ & $\mathrm{N}(4) \# 1-\mathrm{Zn}(1)-\mathrm{N}(1)$ & $114.50(9)$ \\
\hline \multicolumn{4}{|c|}{ Complex 2} \\
\hline $\mathrm{Zn}(1)-\mathrm{O}(3)$ & $1.923(3)$ & $\mathrm{Zn}(1)-\mathrm{N}(1) \# 1$ & $2.005(3)$ \\
\hline $\mathrm{Zn}(1)-\mathrm{O}(1)$ & $1.945(3)$ & $\mathrm{Zn}(1)-\mathrm{N}(4) \# 1$ & $2.007(3)$ \\
\hline $\mathrm{O}(3)-\mathrm{Zn}(1)-\mathrm{O}(1)$ & $101.23(11)$ & $\mathrm{O}(3)-\mathrm{Zn}(1)-\mathrm{N}(1)$ & $118.75(12)$ \\
\hline $\mathrm{O}(3)-\mathrm{Zn}(1)-\mathrm{N}(4) \# 1$ & $102.66(12)$ & $\mathrm{O}(1)-\mathrm{Zn}(1)-\mathrm{N}(1)$ & $106.46(12)$ \\
\hline $\mathrm{O}(1)-\mathrm{Zn}(1)-\mathrm{N}(4) \# 1$ & $115.25(13)$ & $\mathrm{N}(1)-\mathrm{Zn}(1)-\mathrm{N}(4) \# 1$ & $112.38(12)$ \\
\hline \multicolumn{4}{|c|}{ Complex 3} \\
\hline $\mathrm{Cd}(1)-\mathrm{O}(1)$ & $2.266(5)$ & $\mathrm{Cd}(2)-\mathrm{O}(7) \# 3$ & $2.192(5)$ \\
\hline $\mathrm{Cd}(1)-\mathrm{N}(18) \# 1$ & $2.341(5)$ & $\mathrm{Cd}(2)-\mathrm{O}(6)$ & $2.306(5)$ \\
\hline $\mathrm{Cd}(1)-\mathrm{N}(6) \# 2$ & $2.347(6)$ & $\mathrm{Cd}(2)-\mathrm{O}(9)$ & $2.322(6)$ \\
\hline $\mathrm{Cd}(1)-\mathrm{N}(1)$ & $2.348(6)$ & $\mathrm{Cd}(2)-\mathrm{N}(15) \# 4$ & $2.336(6)$ \\
\hline $\mathrm{Cd}(1)-\mathrm{O}(3)$ & $2.419(6)$ & $\mathrm{Cd}(2)-\mathrm{N}(11)$ & $2.353(6)$ \\
\hline $\mathrm{Cd}(1)-\mathrm{O}(4)$ & $2.461(6)$ & $\mathrm{Cd}(2)-\mathrm{O}(5)$ & $2.505(5)$ \\
\hline $\mathrm{O}(1)-\mathrm{Cd}(1)-\mathrm{N}(18) \# 1$ & $142.0(2)$ & $\mathrm{O}(7) \# 3-\mathrm{Cd}(2)-\mathrm{O}(6)$ & $137.7(2)$ \\
\hline $\mathrm{O}(1)-\mathrm{Cd}(1)-\mathrm{N}(6) \# 2$ & $84.0(2)$ & $\mathrm{O}(7) \# 3-\mathrm{Cd}(2)-\mathrm{O}(9)$ & $124.6(2)$ \\
\hline $\mathrm{N}(18) \# 1-\mathrm{Cd}(1)-\mathrm{N}(6) \# 2$ & $91.4(2)$ & $\mathrm{O}(6)-\mathrm{Cd}(2)-\mathrm{O}(9)$ & $95.9(2)$ \\
\hline $\mathrm{O}(1)-\mathrm{Cd}(1)-\mathrm{N}(1)$ & $95.9(2)$ & $\mathrm{O}(7) \# 3-\mathrm{Cd}(2)-\mathrm{N}(15) \# 4$ & $104.6(2)$ \\
\hline $\mathrm{N}(18) \# 1-\mathrm{Cd}(1)-\mathrm{N}(1)$ & $86.2(2)$ & $\mathrm{O}(6)-\mathrm{Cd}(2)-\mathrm{N}(15) \# 4$ & $83.0(2)$ \\
\hline $\mathrm{N}(6) \# 2-\mathrm{Cd}(1)-\mathrm{N}(1)$ & $175.9(2)$ & $\mathrm{O}(9)-\mathrm{Cd}(2)-\mathrm{N}(15) \# 4$ & $92.7(2)$ \\
\hline $\mathrm{O}(1)-\mathrm{Cd}(1)-\mathrm{O}(3)$ & 135.95(19) & $\mathrm{O}(7) \# 3-\mathrm{Cd}(2)-\mathrm{N}(11)$ & $87.2(2)$ \\
\hline $\mathrm{N}(18) \# 1-\mathrm{Cd}(1)-\mathrm{O}(3)$ & $82.0(2)$ & $\mathrm{O}(6)-\mathrm{Cd}(2)-\mathrm{N}(11)$ & $87.8(2)$ \\
\hline $\mathrm{N}(6) \# 2-\mathrm{Cd}(1)-\mathrm{O}(3)$ & $95.7(2)$ & $\mathrm{O}(9)-\mathrm{Cd}(2)-\mathrm{N}(11)$ & $80.9(2)$ \\
\hline $\mathrm{N}(1)-\mathrm{Cd}(1)-\mathrm{O}(3)$ & $87.2(2)$ & $\mathrm{N}(15) \# 4-\mathrm{Cd}(2)-\mathrm{N}(11)$ & $168.2(2)$ \\
\hline $\mathrm{O}(1)-\mathrm{Cd}(1)-\mathrm{O}(4)$ & 83.01(19) & $\mathrm{O}(7) \# 3-\mathrm{Cd}(2)-\mathrm{O}(5)$ & $85.46(19)$ \\
\hline $\mathrm{N}(18) \# 1-\mathrm{Cd}(1)-\mathrm{O}(4)$ & $134.9(2)$ & $\mathrm{O}(6)-\mathrm{Cd}(2)-\mathrm{O}(5)$ & $53.77(18)$ \\
\hline
\end{tabular}




\begin{tabular}{llll}
$\mathrm{N}(6) \# 2-\mathrm{Cd}(1)-\mathrm{O}(4)$ & $98.2(2)$ & $\mathrm{O}(9)-\mathrm{Cd}(2)-\mathrm{O}(5)$ & $149.6(2)$ \\
$\mathrm{N}(1)-\mathrm{Cd}(1)-\mathrm{O}(4)$ & $85.9(2)$ & $\mathrm{N}(15) \# 4-\mathrm{Cd}(2)-\mathrm{O}(5)$ & $82.5(2)$ \\
$\mathrm{O}(3)-\mathrm{Cd}(1)-\mathrm{O}(4)$ & $53.3(2)$ & $\mathrm{N}(11)-\mathrm{Cd}(2)-\mathrm{O}(5)$ & $98.0(2)$ \\
\hline & & $\mathrm{Complex} 4$ & \\
\hline $\mathrm{Cd}(1)-\mathrm{O}(1)$ & $2.272(3)$ & $\mathrm{Cd}(1)-\mathrm{N}(1) \# 1$ & $2.360(3)$ \\
$\mathrm{Cd}(1)-\mathrm{O}(1) \# 1$ & $\mathrm{Cd}(1)-\mathrm{N}(4) \# 2$ & $2.367(3)$ \\
$\mathrm{Cd}(1)-\mathrm{N}(1)$ & $\mathrm{Cd}(1)-\mathrm{N}(4) \# 3$ & $2.367(3)$ \\
$\mathrm{O}(1)-\mathrm{Cd}(1)-\mathrm{O}(1) \# 1$ & $175.0(2)$ & $\mathrm{N}(1)-\mathrm{Cd}(1)-\mathrm{N}(4) \# 2$ & $164.90(11)$ \\
$\mathrm{O}(1)-\mathrm{Cd}(1)-\mathrm{N}(1)$ & $103.02(14)$ & $\mathrm{N}(1) \# 1-\mathrm{Cd}(1)-\mathrm{N}(4) \# 2$ & $84.51(11)$ \\
$\mathrm{O}(1) \# 1-\mathrm{Cd}(1)-\mathrm{N}(1)$ & $80.06(12)$ & $\mathrm{O}(1)-\mathrm{Cd}(1)-\mathrm{N}(4) \# 3$ & $87.42(12)$ \\
$\mathrm{O}(1)-\mathrm{Cd}(1)-\mathrm{N}(1) \# 1$ & $80.06(12)$ & $\mathrm{O}(1) \# 1-\mathrm{Cd}(1)-\mathrm{N}(4) \# 3$ & $88.92(15)$ \\
$\mathrm{O}(1) \# 1-\mathrm{Cd}(1)-\mathrm{N}(1) \# 1$ & $103.02(14)$ & $\mathrm{N}(1)-\mathrm{Cd}(1)-\mathrm{N}(4) \# 3$ & $84.51(11)$ \\
$\mathrm{N}(1)-\mathrm{Cd}(1)-\mathrm{N}(1) \# 1$ & $106.41(15)$ & $\mathrm{N}(1) \# 1-\mathrm{Cd}(1)-\mathrm{N}(4) \# 3$ & $164.90(11)$ \\
$\mathrm{O}(1)-\mathrm{Cd}(1)-\mathrm{N}(4) \# 2$ & $88.92(15)$ & $\mathrm{N}(4) \# 2-\mathrm{Cd}(1)-\mathrm{N}(4) \# 3$ & $86.88(16)$ \\
$\mathrm{O}(1) \# 1-\mathrm{Cd}(1)-\mathrm{N}(4) \# 2$ & $87.42(12)$ & & \\
\hline $\mathrm{Sy}$ & & & \\
\hline
\end{tabular}

Symmetry codes: \#1: $x-1, y-1, z$ for $\mathbf{1}$; $\# 1-x,-y+2,-z$ for 2 ; \#1: $\mathrm{x}, \mathrm{y}+1, \mathrm{z}+1 ; \# 2: \mathrm{x}-1, \mathrm{y}-1, \mathrm{z} ; \# 3: \mathrm{x}-1$, $\mathrm{y}, \mathrm{z}-1 ; \# 4: \mathrm{x}+1, \mathrm{y}+1, \mathrm{z}$ for 3 ; \#1: $-x+1, y,-z+3 / 2 ; \# 2: x+1 / 2, y+1 / 2, z+1 ; \# 3:-x+1 / 2, y+1 / 2,-z+1 / 2$ for 4 .

Table S2. Hydrogen Bonding Distances $(\AA)$ and Angles $\left({ }^{\circ}\right)$ for Complexes 1-3

\begin{tabular}{|c|c|c|c|c|}
\hline $\mathrm{D}-\mathrm{H} \cdots \mathrm{A}$ & $d(D-H)$ & $\mathrm{d}(\mathrm{H} \cdots \mathrm{A})$ & $\mathrm{d}(\mathrm{D} \cdots \mathrm{A})$ & $\angle$ (DHA) \\
\hline \multicolumn{5}{|c|}{ Complex 1} \\
\hline $\mathrm{N}(7)-\mathrm{H}(7 \mathrm{~A}) \cdots \mathrm{O}(4) \# 5$ & 0.86 & 2.065 & 2.918 & 171.22 \\
\hline \multicolumn{5}{|c|}{ Complex 2} \\
\hline $\mathrm{O}(5)-\mathrm{H}(5 \mathrm{~A}) \cdots \mathrm{N}(8) \# 4$ & 0.876 & 2.045 & 2.901 & 165.25 \\
\hline $\mathrm{O}(5)-\mathrm{H}(5 \mathrm{~B}) \cdots \mathrm{O}(4) \# 5$ & 0.875 & 1.958 & 2.785 & 157.16 \\
\hline \multicolumn{5}{|c|}{ Complex 3} \\
\hline $\mathrm{O}(10)-\mathrm{H}(10 \mathrm{~A}) \cdots \mathrm{O}(1)$ & 0.850 & 2.506 & 2.999 & 117.84 \\
\hline
\end{tabular}




$\begin{array}{lllll}\mathrm{O}(10)-\mathrm{H}(10 \mathrm{~B}) \cdots \mathrm{O}(5) & 0.850 & 2.175 & 2.816 & 132.00 \\ \mathrm{O}(11)-\mathrm{H}(11 \mathrm{~A}) \cdots \mathrm{O}(4) \# 3 & 0.850 & 2.212 & 2.906 & 138.36\end{array}$

Symmetry codes: $\# 5:-x,-y+1,-z$ for $\mathbf{1} ; \# 4:-x+2,-y+1,-z+1 ; \# 5:-x+1,-y+1,-z$ for $2 ; \# 3: \mathrm{x}-1, \mathrm{y}$, $\mathrm{z}-1$ for 3 .

Table S3. Crystal Structure Affected by Organic Ligands in Complexes 1-4

\begin{tabular}{|c|c|c|c|c|c|}
\hline complexes & $\begin{array}{c}\text { Connectivity } \\
\text { of } \\
\text { BPTA/TTPA/ } \\
\text { TTPBDA }\end{array}$ & $\begin{array}{c}\text { Connectivity } \\
\text { of } \mathbf{H}_{2} \text { TPA }\end{array}$ & dimensions & topology & interpenetration \\
\hline $\mathbf{1}$ & 2 & 2 & $2 \mathrm{D}$ & sql & $\begin{array}{c}\text { 3-fold } \\
\text { interpenetration }\end{array}$ \\
\hline $\mathbf{2}$ & 2 & 2 & $2 \mathrm{D}$ & $\mathbf{h c b}$ & $\begin{array}{c}\text { 4-fold } \\
\text { interpenetration } \\
\text { (considering } \\
\text { hydrogen bonds) }\end{array}$ \\
\hline $\mathbf{3}$ & $3 / 2$ & 2 & 3D & $\mathbf{3 , 4 , 5 T 8 6}$ & $\begin{array}{c}\text { 4-fold } \\
\text { interpenetration }\end{array}$ \\
\hline $\mathbf{4}$ & 4 & 2 & 3D & new topology & self-penetration \\
\hline
\end{tabular}

Table S4. Photoluminescence Data for Organic Ligands, Complexes 1-4 and 1'-4'

\begin{tabular}{|c|c|c|c|c|c|}
\hline Complexes & $\lambda_{\text {ex }}(\mathrm{nm})$ & $\lambda_{\mathrm{em}}(\mathrm{nm})$ & Ligand & $\lambda_{\text {ex }}(\mathbf{n m})$ & $\lambda_{\mathrm{em}}(\mathrm{nm})$ \\
\hline 1 & \multirow{2}{*}{397} & 420 & $\mathrm{H}_{2} \mathrm{BPA}$ & 362 & 417 \\
\hline $\mathbf{1}^{\prime}$ & & 434 & BTPA & 363 & 396 \\
\hline 2 & \multirow{2}{*}{369} & 407 & TTPA & 388 & 416 \\
\hline $2^{\prime}$ & & 410 & TTPBDA & 404 & 430 \\
\hline 3 & \multirow{2}{*}{380} & 413 & & & \\
\hline $3^{\prime}$ & & 405 & & & \\
\hline 4 & \multirow{2}{*}{397} & 428 & & & \\
\hline $4^{\prime}$ & & 431 & & & \\
\hline
\end{tabular}

\section{Reference}

(S1) Shi, Z. Z.; Pan, Z. R.; Zhang C. L.; Zheng, H. G. Dalton Trans. 2015, 44, 16854-16864. 
(S2) Yao, X. Q.; Cao, D. P.; Hu, J. S.; Li, Y. Z.; Guo, Z. J.; Zheng, H. G. Cryst. Growth Des. 2011, $11,231-239$.

(S3) Ishow, E.; Brosseau, A.; Clavier, G.; Nakatani, K.; Pansu, R. B.; Vachon, J. J.; Tauc, P.; Chauvat, D.; Mendonça, C. R.; Piovesan, E. J. Am. Chem. Soc. 2007, 129, 8970-8971.

(S4) Qin, L.; Zhang, M. D.; Yang, Q. X.; Li, Y. Z.; Zheng, H. G. Cryst. Growth Des. 2013, 13, $5045-5049$.

(S5) Bruker 2000, SMART (Version 5.0), SAINT-plus (Version 6), SHELXTL(Version 6.1), and SADABS (Version 2.03); Bruker AXS Inc.: Madison, WI. 